Published in final edited form as:

Nat Med. 2018 February ; 24(2): 176-185. doi:10.1038/nm.4475.

\title{
Positively selected enhancer elements endow osteosarcoma cells with metastatic competence
}

\author{
James J. Morrow ${ }^{1,2}$, lan Bayles ${ }^{2}$, Alister PW Funnell ${ }^{3}$, Tyler E. Miller ${ }^{1}$, Alina Saiakhova ${ }^{2}$, \\ Michael M. Lizardo ${ }^{4}$, Cynthia F. Bartels ${ }^{2}$, Maaike Y. Kapteijn ${ }^{5}$, Stevephen Hung ${ }^{2}$, Arnulfo \\ Mendoza $^{4}$, Gursimran Dhillon ${ }^{2}$, Daniel R. Chee ${ }^{6}$, Jay T. Myers ${ }^{7}$, Frederick Allen ${ }^{1}$, Marco \\ Gambarotti $^{8}$, Alberto Righi ${ }^{8}$, Analisa DiFeo ${ }^{9}$, Brian P. Rubin ${ }^{10}$, Alex Y. Huang ${ }^{1,7}$, Paul S. \\ Meltzer $^{11}$, Lee J. Helman ${ }^{4}$, Piero Picci ${ }^{8}$, Henri Versteeg ${ }^{5}$, John Stamatoyannopolus ${ }^{3}$, Chand \\ Khanna ${ }^{4,}{ }^{*}$, and Peter C. Scacheri ${ }^{2,8,{ }^{* \star}}$
}

\begin{abstract}
${ }^{1}$ Department of Pathology, Case Western Reserve University, Cleveland, OH 44106, USA
${ }^{2}$ Department of Genetics and Genome Sciences, Case Western Reserve University, Cleveland, $\mathrm{OH} 44106$, USA ${ }^{3}$ Altius Institute for Biomedical Sciences, Seattle, Washington, USA ${ }^{4}$ Pediatric Oncology Branch, Center for Cancer Research, NCI, NIH, Bethesda, MD, 20892 USA ${ }^{5}$ Thrombosis and Hemostasis Division, Department of Internal Medicine, LUMC, Leiden, Netherlands ${ }^{6}$ Department of Genome Sciences, University of Washington, Seattle, Washington 98195, USA ${ }^{7}$ Department of Pediatrics, Case Western Reserve University, Cleveland, $\mathrm{OH} 44106$, USA ${ }^{8}$ Research Laboratory, Istituto Ortopedico Rizzoli, Via Pupilli 1, 40136, Bologna, Italy ${ }^{9}$ Case Comprehensive Cancer Center, Case Western Reserve University, Cleveland, $\mathrm{OH}$ 44106, USA ${ }^{10}$ Departments of Anatomic Pathology and Molecular Genetics, Cleveland Clinic, Lerner Research Institute and Taussig Cancer Center, Cleveland, OH 44195, USA ${ }^{11}$ Genetics Branch, Center for Cancer Research, NCI, NIH, Bethesda, MD, 20892 USA
\end{abstract}

\begin{abstract}
Metastasis results from a complex set of traits acquired by tumor cells, distinct from those necessary for tumorigenesis. Here, we investigate the contribution of enhancer elements to the metastatic phenotype of osteosarcoma. Through epigenomic profiling, we identify substantial
\end{abstract}

\footnotetext{
Users may view, print, copy, and download text and data-mine the content in such documents, for the purposes of academic research, subject always to the full Conditions of use: http://www.nature.com/authors/editorial_policies/license.html\#terms

${ }^{* *}$ Correspondence: peter.scacheri@ case.edu.

*C. Khanna is currently with Ethos Discovery, Washington DC and Ethos Veterinary Health, Woburn, MA.

Contributions

J.J.M., C.K., and P.C.S. conceived of the overall experimental design. J.J.M., C.F.B., and G.D. generated ChIP-seq, RNA-seq, and DHS-seq data. J.J.M., A.S., S.H., and P.C.S. completed analysis of ChIP-seq, RNA-seq, and DHS-seq data. J.J.M. and T.E.M. designed and completed shRNA screening experiment and analysis. T.E.M. completed functional enrichment analysis of RNA-seq data. J.J.M. and I.B. generated 4C-seq data. J.J.M. and A.S. analyzed 4C-seq data. J.J.M., A.M., and I.B. completed in vivo and ex vivo metastasis experiments. J.J.M., J.T.M., and F.A. designed and completed orthotopic metastasis experiments. J.J.M., D.R.C., and A.P.W.F. designed and completed TALEN deletion experiments. M.Y.K. completed in vitro F3 experiments. M.G., A.R., and P.P. provided patient tumor samples and clinical data. B.P.R. assessed F3 staining in patient tissue microarray. A.D., A.Y.H., P.S.M., L.J.H., H.V., J.S., C.K., and P.C.S. provided technical expertise and facilities to complete experiments. J.J.M. and P.C.S. analyzed all data and wrote the paper. All authors provided intellectual input, edited, and approved the final manuscript.

Data Availability

The datasets generated during and/or analysed during the current study are available in the GEO repository under the following accession number: GEO74230.
} 
differences in enhancer activity between primary and metastatic tumors in human patients as well as near-isogenic pairs of high and low lung-metastatic osteosarcoma cells. We term these regions Metastatic Variant Enhancer Loci (Met-VELs). Met-VELs drive coordinated waves of gene expression during metastatic colonization of the lung. Met-VELs cluster non-randomly in the genome, indicating that activity of these enhancers and their associated gene targets are positively selected. As evidence of this causal association, osteosarcoma lung metastasis is inhibited by global interruptions of Met-VEL-associated gene expression via pharmacologic BET inhibition, by knockdown of AP-1 transcription factors that occupy Met-VELs, and by knockdown or functional inhibition of individual genes activated by Met-VELs, such as coagulation factor III/ tissue factor (F3). We further show that genetic deletion of a single Met-VEL at the F3 locus blocks metastatic cell outgrowth in the lung. These findings indicate that Met-VELs and the genes they regulate play a functional role in metastasis and may be suitable targets for anti-metastatic therapies.

\section{Introduction}

More than $90 \%$ of all cancer deaths are the result of tumor metastasis ${ }^{1}$. The physical process of tumor cell dissemination and metastatic colonization of distant secondary sites has been well described ${ }^{2}$. Whole genome sequencing studies have elucidated the evolutionary phylogeny of metastatic dissemination ${ }^{3,4}$, and gene expression studies have revealed many of the genes that mediate the progressive steps of metastasis and drive organ-specific colonization $^{5-7}$. These studies suggest that adaptation of metastatic tumor cells to the microenvironments of their destination organs is accompanied by a shift in cell state through widespread changes in the transcriptional output of metastatic cell genomes. Whether the shift is driven by genetic or epigenetic factors, or a combination of both of these mechanisms is not yet clear.

During normal development, gene expression changes that accompany cell state transitions are driven by altered activity of gene enhancer elements ${ }^{8-10}$. Enhancers govern cell typespecific expression programs and are defined by signature chromatin features including H3K4me1, H3K27ac, and DNase hypersensitivity ${ }^{11}$. Enhancers appear to be important in tumorigenesis as well. Previous studies have demonstrated that malignant transformation is accompanied by locus-specific gains and losses in enhancer activity across the epigenome, termed Variant Enhancer Loci (VELs) ${ }^{12,13}$. Others have shown that in many types of cancers, clusters of active enhancers called super-enhancers (SEs) mediate dysregulated expression of oncogenes ${ }^{14,15}$. Collectively, these studies suggest that aberrant enhancer activity is a key driver of tumor formation and maintenance.

Altered transcriptional programs play a role in metastatic tumor progression. In certain model systems, these transcriptional programs have been associated with metastatic colonization of specific secondary organs ${ }^{5-7,16}$. Recently, epigenetic changes have been associated with transcriptional changes during metastasis ${ }^{17}$. However, the contribution of gene enhancers to metastatic transcription is not well understood. Based on the knowledge that enhancers drive cell-state transitions during normal development and tumorigenesis, we 
hypothesized that enhancers may play a similar role in the transition of cancer cells from one developmentally distinct tissue to another during metastatic progression.

Osteosarcoma is the most common primary malignancy of the bone with peak incidence in children and adolescents. Clinical outcomes for patients have not improved for 30 years and there are currently no approved targeted anti-metastatic therapies for osteosarcoma in wide clinical use ${ }^{18}$. More than $75 \%$ of osteosarcoma metastases occur at the secondary site of the lung, which is the cause of the overwhelming majority of osteosarcoma related deaths ${ }^{19}$. In this study, we leverage the knowledge that gene enhancer activity is the cornerstone of cellular phenotypes and cell type specific gene expression ${ }^{9,20}$ to gain new insight into the regulatory mechanisms that allow metastatic osteosarcoma cells to overcome the barriers to colonization encountered as these cells engage the lung microenvironment. Our studies establish that enhancer elements endow tumor cells with metastatic capacity and that targeted inhibition of genes associated with enhancer alterations, or deletion of altered enhancers themselves is sufficient to block metastatic colonization and proliferation.

\section{Results}

\section{The Metastatic Phenotype of Human Osteosarcoma is Associated with Variant Enhancer Loci}

We mapped the locations of putative enhancer elements genome wide through ChIP-seq of the canonical enhancer-histone marks, $\mathrm{H} 3 \mathrm{~K} 4 \mathrm{me} 1$ and $\mathrm{H} 3 \mathrm{~K} 27 \mathrm{ac}$ in matched primary tumors and lung metastases from five osteosarcoma patients. We also performed H3K4me1 and H3K27ac ChIP-seq, and DNase-seq on a panel of five well-characterized ${ }^{21}$ metastatic and non-metastatic human osteosarcoma cell line pairs representing three distinct mechanisms of metastatic derivation including in vivo selection, treatment with a mutagenic compound, and introduction of an oncogenic driver (Fig. 1a). Based on the previous finding that H3K4me1 broadly correlates with both poised and active enhancers ${ }^{22,23}$, we used this histone mark for our initial comparisons.

We found thousands of regions where H3K4me1 signals showed at least a 3-fold difference in enrichment between conditions (Fig. 1b, 1c). The metastasis-associated gains and losses of the H3K4me1 signal were reminiscent of those that we previously identified in the setting of primary tumor development through comparisons of primary colon tumors and normal colon tissue, known as VELs ${ }^{12,13}$. In distinction, we now and herein term the regions that show differential enrichment of $\mathrm{H} 3 \mathrm{~K} 4 \mathrm{me} 1$ between metastatic samples and non-metastatic controls Metastatic Variant Enhancer Loci, or Met-VELs. Enhancers defined by differential enrichment of H3K4me1 generally showed concordant changes in H3K27ac ChIP-seq signals and DNase-seq signals (Fig. 1d and Extended Data Fig. 1), indicating robust commissioning and decommissioning of active enhancer elements at these loci. Across all samples, we found that on average $9.3 \%$ of all enhancers in a given metastatic cell line or tumor were gained relative to controls while $16.4 \%$ of enhancers present in non-metastatic cell lines or primary tumors were lost (Fig. 1e).

We next assessed the degree of Met-VEL heterogeneity across the cohort (Fig. 1F). MetVELs were more concordant between two metastatic cell lines (MNNG and 143B, labeled 
with asterisks) derived from a single parental cell line (HOS) than those derived from distinct parental cell populations ( $40.8 \%$ versus $0.2-9.3 \%, \mathrm{P}<0.001)$, suggesting that the specific enhancer elements that undergo activation and silencing is non-random and may be in part driven by the genetic and/or epigenetic makeup of the parental cell line. Met-VELs were heterogeneous among the remaining samples, with $23-69.1 \%$ showing overlap with at least one other sample, and 4.2-18.4\% showing overlap with 2 or more samples. No MetVELs were common to all cell lines or tissue samples. The heterogeneity observed could be a result of different selective pressures between the experimental approaches used to derive the metastatic cell lines, and also heterogeneity in the selective process of metastasis in patients. Additionally, the genetic heterogeneity among primary osteosarcoma tumors may contribute to the epigenetic heterogeneity we observe in cell lines and tumors.

An initial survey of Met-VEL distributions revealed dense clusters at distinct regions across the epigenome often in the vicinity of individual genes, similar to super-enhancers (Extended Data Fig. 2a). This finding led us to hypothesize that enhancer activity in these regions was non-randomly acquired due to selective pressures incurred during the process of metastatic progression. We systematically tested this hypothesis and found numerous loci with Met-VEL counts significantly greater than expected by chance in all samples (clusters in exemplar pairs displayed in Fig. 1g and Extended Data Fig. 2b). Several of the genes associated with Met-VEL clusters in both primary human samples and in the cell lines have been previously implicated in tumor biology and/or progression. ANGPT1 is a TIE2 receptor agonist that plays a crucial role in angiogenesis and is currently being studied as a therapeutic target in malignancy ${ }^{24}$. Growth hormone receptor $(G H R)^{25,26}$, phosphodiesterase 10A $(P D E 10 A)^{27}$, and tissue factor $(F 3)$, have all been previously implicated in tumor biology and/or progression. F3 is a well-described activator of normal blood coagulation. In the setting of cancer, F3 plays tumor-cell-endogenous roles in promoting tumor growth and metastasis in multiple cancers, but the mechanism underlying its activation is not fully defined ${ }^{28}$. All metastatic/non-metastatic cell line and primary/ metastatic tumor pairs studied showed evidence of non-random acquisition and loss of enhancer clusters (Extended Data Fig. 2c). On average across the cohort, 22\% of all MetVELs were found to reside in Met-VEL clusters (Extended Data Fig. 2d).

\section{Metastatic Variant Enhancer Loci (Met-VELs) Dynamically Modulate Gene Expression as Tumor Cells Engage the Lung Microenvironment}

To investigate the role of Met-VELs in modulating gene expression during metastasis, we utilized an ex vivo mouse model of osteosarcoma lung metastasis ${ }^{29}$. In this model, we seed GFP-expressing tumor cells to the lungs of mice by intravenous injection and culture lung sections ex vivo. This approach allows us to track metastatic outgrowth of GFP-labeled tumor cells in real time and to assess dynamic changes in gene expression (Fig. 2a). We performed RNA-seq at both early (24-hours) and late (day 14) time points in three cell line pairs. We associated Met-VELs with predicted target genes using PreSTIGE ${ }^{30}$ and validated that $>94.5 \%$ of predicted Met-VEL gene targets lie within the same topologically associating domains (TADs) as the corresponding enhancer. In all cases, genes associated with gained Met-VELs and Met-VEL clusters were generally expressed at higher levels in metastatic cells within the lung microenvironment than in their corresponding non-metastatic parental 
cell lines while genes associated with lost Met-VELs and lost Met-VEL clusters were expressed at lower levels (Fig. 2b and Extended Data Fig. 3a, b). To investigate whether Met-VEL-associated gene sets represent a transcriptional program specifically modulated in the setting of metastasis, we compared expression across conditions. The degree of differential expression of Met-VEL associated genes in the parental vs. metastatic cells was greater in ex vivo lung culture than in standard in vitro culture conditions (Extended Data Fig. 4a, b) indicating that modulation of these transcriptional programs represents a cellular response to external cues from the lung microenvironment. Met-VEL associated gene sets showed little overlap $(<27 \%)$ with the most differentially expressed genes in each metastatic/non-metastatic cell line pair indicating that Met-VEL associated genes represent a distinct set from those likely to be identified by expression data alone (Extended Data Fig. $4 c, d)$.

Subsets of genes associated with gained Met-VELs became highly expressed within 24hrs of arrival of metastatic cells to the lung, others were only activated later during metastatic outgrowth, and a third subset were constitutively up-regulated (Fig. 2c and Extended Data Fig. 3c). We assessed these gene sets for functional enrichment, and found that the phasic waves of gene expression coordinated by Met-VELs during lung colonization are associated with distinct cellular functions (Fig. $2 \mathrm{~d}$ and Extended Data Fig. 3e). We verified that gained Met-VEL genes upregulated in the ex vivo lung model are frequently elevated in osteosarcoma patient lung metastases relative to primary tumors (Fig. 2e) and many of the same gene sets are enriched in gained Met-VEL target genes in osteosarcoma lung metastases in patients (Extended Data Fig. 5), thereby verifying that the genes identified using the cell line models and the ex vivo approach are representative of those dysregulated in human patients.

We performed motif enrichment analysis and identified a number of commonly expressed TFs with enriched motifs in gained and lost Met-VELs across all three pairs analyzed. The most highly enriched motifs include many members of the AP-1 complex (JUN, JUNB, JUND, FOS, and FOSL1) (Fig. 2f and Extended Data Fig. 3d) which has been previously shown to play a key role in osteosarcoma metastasis ${ }^{31}$. Intriguingly, AP-1 motifs were enriched at both gained and lost Met-VELs. This finding suggests that Met-VELs likely alter the transcriptional programs mediated by AP-1 during osteosarcoma metastasis. We verified that FOS and FOSL1 are bound at gained Met-VELs by ChIP-seq (Fig. 2g).

\section{Met-VEL Associated Gene Expression is Required for Metastatic Colonization}

Based on the finding that gained Met-VELs have high levels of the activating histone mark $\mathrm{H} 3 \mathrm{~K} 27 \mathrm{ac}$ and the knowledge that the BET-family protein, BRD4, is critical for transcriptional activation by $\mathrm{H} 3 \mathrm{~K} 27 \mathrm{ac}-$ marked enhancers, we reasoned that BRD4 inhibition may interrupt Met-VEL gene expression and indeed metastasis. To test this hypothesis, we used the BRD4-inhibitor JQ1, which displaces BRD4 from H3K27ac-marked enhancers ${ }^{15,32}$. JQ1 has been shown to inhibit osteosarcoma primary tumor formation through its effects on both tumor cells and bone cells within the tumor microenvironment ${ }^{33}$. We found that JQ1 showed potent anti-proliferative effects on metastatic tumor cells growing in the lung microenvironment without affecting the surrounding normal lung tissue 
(Extended Data Fig. 6a-c). The anti-proliferative effects were associated with selective suppression of gained Met-VEL target genes that are normally up-regulated in metastatic cells in response to cues from the lung microenvironment (Extended Data Fig. 6d, e). Intriguingly, JQ1 more potently suppressed gained Met-VEL genes than genes associated with super-enhancers (Extended Data Fig. 6f) that have previously been linked to JQ1's antitumor properties in other tumor models ${ }^{15,34,35}$.

The results of the JQ1 studies suggest that activation of gained Met-VEL genes is necessary for metastatic outgrowth. To further test this hypothesis, we conducted a functional in vivo RNAi assay. We constructed a custom shRNA library targeting 33 genes. This gene list included 20 genes associated with gained Met-VELs or Met-VEL clusters, 11 TFs with motifs enriched at Met-VELs, and 2 genes of interest from other ongoing studies. We cloned the shRNA library into a tetracycline-inducible lentiviral construct (LTREPIR, Fig. 3a) modified from a similar construct previously published ${ }^{36}$. Using a DsRed fluorescent reporter of shRNA induction, we show that this construct was robustly induced upon exposure to doxycycline and not leaky in the absence of doxycycline (Extended Data Fig. 7). We conducted parallel screens in vivo and in vitro to allow us to distinguish genes that specifically inhibit metastatic outgrowth in the in vivo microenvironment, or metastasis dependency genes, from genes whose inhibition reduces cellular growth independent of context (Fig. 3b). In the in vivo screen, transduced cells were delivered via tail vein injection into mice pre-treated with doxycycline. Mice were maintained on doxycycline throughout the 21-day course of the experiment. In the parallel in vitro screen, transduced cells were treated in culture with doxycycline for 21 days. At the conclusion of the experiment, induced cells actively expressing shRNA (DsRed+/GFP+) were sorted from mouse lungs or in vitro culture by FACS. DNA was isolated from these cells, along with uninduced cells from the initial population (input), and shRNAs were amplified, sequenced, and aligned to the reference shRNA sequences of the library to determine normalized representation of each shRNA.

We defined metastasis dependency genes as those whose knockdown inhibited in vivo metastasis significantly more than in vitro growth. First, shRNA representations in metastatic tumor cells were compared to input representations to identify shRNAs depleted from the population of cells during metastatic outgrowth in the lung (Fig. 3c). Genes that were targets of at least two unique shRNAs (the pool contained 3-4 shRNAs per gene) that inhibited metastatic outgrowth to a greater degree than all negative controls were defined as initial hits. Because we used a second filter for these hits in this screen and planned further functional validation experiments, we intentionally chose a relatively inclusive threshold for initial hit calling. We found that 13 of 33 genes (39\%) included in our screen met this criterion. To determine if depletion of cells expressing these shRNAs was specific to metastasis, we compared the relative representations of hits in the in vivo induced population of cells to in vitro induced controls (Fig. 3d). Genes whose shRNAs were significantly more depleted in vivo than in vitro were considered metastasis dependency genes. 6 of the 13 initial hits met this criterion (Extended Data Table 1). Metastasis dependency genes included four genes associated with gained Met-VEL clusters ( $F 3, F B X O 42, F L N A$, and FOXO3) as well as two AP-1 complex TFs whose motifs are enriched in Met-VELs and were shown to bind at these enhancers (FOS and FOSL1). These results indicate that metastatic 
colonization of the lung by osteosarcoma cells is dependent on expression of a subset of individual genes associated with gained Met-VEL clusters as well as AP-1 complex TFs likely to regulate Met-VEL transcriptional programs.

Among the Met-VEL genes, $F 3$ emerged as a top candidate driver of metastasis in osteosarcoma. In the MG63.3 cell line, F3 was associated with a gained Met-VEL cluster containing the second highest gained Met-VEL count of the entire data set (Fig. 1g), suggesting that this locus was under particularly strong positive selection during metastatic derivation. Gained Met-VELs in the F3 cluster also showed higher levels of H3K27ac and DNase accessibility in MG63.3 cells compared to the parental MG63 cell line and chromatin conformation capture studies confirmed that these enhancers physically contact the transcription start site of $F 3$ (Extended Data Fig. 8a). F3 was more highly expressed in MG63.3 cells during metastatic outgrowth than in the parental MG63 cells (Extended Data Fig. 8b). In addition, two other metastatic cell lines, MNNG and 143B, showed active enhancer signals at the $F 3$ locus, similar to MG63.3 (Extended Data Fig. 8d) and expressed $F 3$ at higher levels during metastatic outgrowth than their parental cell line (Extended Data Fig. 8b). To verify that elevated $F 3$ transcript levels were recapitulated at the protein level and also not an artifact of ex vivo culture, we performed immunofluorescence analysis of lung metastases from a fully in vivo model of metastasis and confirmed that metastatic osteosarcoma cells expressed higher levels of F3 protein than non-metastatic cells (Fig. 4a, b). Quantification of $F 3$ levels directly in human osteosarcoma patient samples showed that F3 was elevated in lung metastases relative to primary tumors (Extended Data Fig. 8c). Using a tissue microarray, we confirmed that $\mathrm{F} 3$ protein was highly expressed in lung metastases from human osteosarcoma patients. F3 was expressed in $>50 \%$ of tumor cells in 18/18 lung metastases and F3 showed strongly positive staining in 17/18 samples (Fig. 4c, d, Extended Data Fig 9).

To determine whether F3 upregulation in osteosarcoma lung metastases was a result of enhancer dysregulation, we analyzed the enhancer epigenomes of 10 pairs of patientmatched primary and metastatic tumors. While we do not observe de novo creation of a sufficient number of gained Met-VELs at the $F 3$ locus to meet criteria to be called a gained cluster, the locus has an enhancer cluster that in 9 out of 10 metastatic samples is called as a super-enhancer and ranks among the most enriched H3K27ac sites (Extended Data Fig 10a). Comparison of the super-enhancer landscapes across all samples showed that the $F 3$ superenhancer was among the top $1.3 \%$ of super-enhancers enriched in lung metastases relative to primary tumors $(\mathrm{P}<0.05)$. This was true when metastatic tumors were compared to primary tumors alone and when metastatic/non-metastatic cell lines were included in the analysis (Extended Data Fig 10b).

We further interrogated the enhancer profiles of these 10 paired samples and verified that common Met-VEL gene targets in cell lines often overlapped with common Met-VEL targets in primary patient samples (Table S2).

We next tested the functional contribution of $\mathrm{F} 3$ expression to the metastatic phenotype. We cloned two shRNAs targeting $F 3$ that were not included in the RNAi assay into the tetracycline-inducible LTREPIR construct. Relative to uninduced control cells, F3 
expression was reduced 44-63\% 40hrs after induction of each shRNA (Extended Data Fig. 11). F3 knockdown with these shRNAs did not affect the in vitro growth rate of metastatic MG63.3 or MNNG cells (Extended Data Fig. 12a), but significantly reduced metastatic outgrowth of these cells in ex vivo lung culture (Extended Data Fig. 12b, c), supporting the metastasis-specific role for F3 in this setting. F3 knockdown also significantly reduced metastatic outgrowth of osteosarcoma cells in vivo (Extended Data Fig. 12d, e) and substantially prolonged survival of mice injected with metastatic osteosarcoma cells (Fig. 4e). To further test whether F3 knockdown reduces in vivo growth of metastatic cells generally or if this effect is specific to metastatic outgrowth of cells in the lung, we completed a spontaneous metastasis experiment using an orthotopic injection model. We found that F3 knockdown did not reduce primary tumor development or growth (Fig. 4f), but significantly inhibited metastasis, reducing average metastatic burden by 3.6 fold (Fig. 4g, h). While extensive GFP+ metastatic lesions were observed in control mice, lungs of mice in the F3 knockdown group were virtually devoid of metastatic lesions with only rare single GFP+ cells observable in most cases (Extended Data Fig. 13).

F3 is known to both induce blood coagulation by mediating the generation of the active form of factor X (FXa) and to promote cell survival and proliferation upon binding to activated factor VII via intracellular signaling mechanisms ${ }^{28}$. To determine the relative contributions of each of these functions to the pro-metastatic role of F3 we used monoclonal antibodies generated to inhibit each of these functions independently ${ }^{37}$. As expected, we found that MG63.3 cells produce more FXa in in vitro assays than MG63 cells (Fig. 4i). We confirmed that anti-coagulant Mab-5G9 robustly inhibited this activity while Mab-10H10, designed to prevent intracellular signaling, did not. We next tested the anti-metastatic effects of these antibodies in vivo. We co-injected MG63.3 cells with each of these antibodies or IgG control into the tail veins of mice and found that both inhibited metastasis, with Mab-5G9 showing a more pronounced effect (Fig. $4 \mathrm{j}, \mathrm{k})$. These results indicate that both the intracellular signaling and pro-coagulant functions of $\mathrm{F} 3$ contribute to metastatic progression, but that F3's pro-coagulant activity is especially critical to metastatic success. Collectively, these results suggest that Met-VELs regulate expression of genes, such as F3, with critical functions during metastatic progression.

To directly test the role of Met-VELs in mediating the metastatic phenotype we employed transcription activator-like effector nuclease (TALEN) genome editing to excise one of the gained Met-VELs predicted to regulate F3 in the metastatic cells. We targeted a Met-VEL located in a particularly robust DHS site containing high levels of both H3K4me1 and H3K27ac (Fig. 5a). This site also showed high ChIP-seq enrichment of both FOS and FOSL1 AP-1 complex members. We generated a cell clone with homozygous deletion of this Met-VEL, verified by Sanger sequencing (Fig 5a). Edited and unedited control cells were then seeded to mouse lungs via tail vein injection and the growth of the cells was monitored in the lungs using the ex vivo metastasis assay. Quantification of F3 levels 24hours post-injection showed that F3 expression was reduced by $34 \%$ in the edited cells relative to unedited control cells (Fig. 5b, c). By day 5, lungs seeded with the F3 Met-VELedited cells were nearly devoid of tumor cells, while extensive GFP+ metastatic lesions were observed in lungs seeded with the unedited cells (Fig. 5d). Quantification showed that deletion of this gained Met-VEL decreased metastatic burden by $78 \%$ (Fig. 5e). 


\section{Discussion}

While many of the genes responsible for metastatic progression have been identified across tumor types, the underlying mechanisms regulating expression of these genes are not well defined. Our studies demonstrate that altered enhancer activity is a fundamental mechanism by which tumor cells regulate gene expression during the dynamic process of metastasis, and thereby acquire metastatic traits. Through epigenomic profiling experiments, we identify enhancers that distinguish human osteosarcoma lung metastases from matched primary tumors and verify that these differences are also present in near-isogenic metastatic and nonmetastatic paired human osteosarcoma cell lines. Subsets of these enhancer changes occur in non-random clusters indicating that they were positively selected during the process of metastatic derivation. These results demonstrate that the metastatic phenotype is accompanied by a shift in the enhancer epigenome, similar to the enhancer shifts that occur as cells transition through successive stages of embryonic development ${ }^{8-10}$, or during conversion of a normal cell to the malignant state $\mathrm{e}^{12,14,15}$. The findings suggest that the evolutionary selective forces encountered by tumor cells during metastasis act to shape the enhancer landscape of metastatically successful cancer cell populations. The result of this selection is a population of cells possessing all of the traits necessary to overcome the barriers to metastatic colonization at distant tissues. Indeed, we show that many genes previously associated with metastasis become dysregulated through alterations in enhancer activity.

We provide multiple lines of evidence that acquired enhancer changes in metastatic osteosarcoma cells are functional and relevant to the osteosarcoma metastatic phenotype in experimental models and human tissues. First, we show that Met-VEL genes are dynamically regulated as metastatic cells engage the lung microenvironment and proliferate. Second, we demonstrate that metastatic cell outgrowth in the lung can be mitigated with through BET inhibition, and that this effect is associated with selective suppression of genes that are normally activated by Met-VELs in the lung. Third, through in vivo functional RNAi-based assays, we demonstrate that the metastatic capacity of the osteosarcoma cells can be diminished by targeted inhibition of individual Met-VEL genes and associated AP1family transcription factors that likely regulate Met-VEL transcriptional programs. Using a fully in vivo spontaneous model of metastasis, we further verify that one such Met-VEL gene, Tissue Factor (F3), is a clinically relevant, bone fide metastasis dependency gene essential for metastatic colonization with no apparent advantage to growth of the primary tumor. Interrupting the signaling and pro-coagulant functions of F3 was sufficient to inhibit metastasis, shedding light on the biological role of this gene in the metastatic progression of osteosarcoma. Our genomic Met-VEL deletion experiments demonstrate that the loss of function of a single gained enhancer at the $F 3$ locus is sufficient to impair metastatic colonization and subsequent outgrowth in mice, indicating that enhancer activation contributed to acquisition of the metastatic phenotype of these cells.

Our current model is that F3 upregulation via the aberrant activation of its enhancers is required for lung colonization by metastatic osteosarcoma cells. It is not yet clear whether neutralizing F3 after colonization would lead to regression of overt metastases. The current standard of care for osteosarcoma involves multiple cycles of neoadjuvant combination 
chemotherapy before surgical removal of the primary tumor ${ }^{18}$. As such, there is a period of several months between the time of diagnosis and primary tumor control. This treatment period is critical as there is the continued potential for cells to leave the primary tumor and seed the lungs. We believe that the most clear therapeutic setting for F3 inhibition would be during this period of neoadjuvant therapy. The findings presented here may present a rationale for testing F3 inhibition in such a setting. Further studies are required to determine whether there may be clinical benefit from continued F3 inhibition after primary tumor control is achieved.

Collectively our findings indicate that altered enhancer activity is a driver of gene expression that is critical for tumor cells to overcome the barriers of distant tissue colonization during metastasis. It is well established that primary tumor formation is driven by a combination of genetic and epigenetic events ${ }^{38}$. With respect to metastasis, studies have shown that primary and matched metastatic tumors are broadly similar at the genetic level with no recurrent mutations identified in metastases that were not present in the primary tumor ${ }^{3,4,39-44}$. These studies suggest that primary tumors are likely already genetically equipped with the ability to metastasize. Further this implies that epigenetic processes may mediate the shift in cell state that accompanies metastatic progression, as proposed by others ${ }^{17,45-48}$. Consistent with this epigenetic hypothesis, we show that osteosarcoma metastasis is accompanied by a shift in epigenetic state at enhancer elements. While our findings are not mutually exclusive with genetic theories of metastatic progression, we find that positive selection of enhancer activity is a fundamental component of the metastatic phenotype.

Our findings add to growing evidence implicating epigenomic processes in metastasis. McDonald et al. recently showed that pancreatic cancer metastasis is associated with widespread changes in heterochromatin defined by $\mathrm{H} 3 \mathrm{~K} 9 / \mathrm{H} 4 \mathrm{~K} 20$ methylation ${ }^{17}$. Denny et al. showed that differences in chromatin accessibility correlated with metastatic progression in NFIB-driven small cell lung cancer ${ }^{49}$. Most recently, Roe et al. showed that aberrant enhancer activity mediated by FOXA1 promotes metastasis in pancreatic cancer ${ }^{50}$. Collectively, these studies demonstrate that chromatin changes drive the metastatic phenotype across various cancers. Our findings are well aligned with these studies, but further implicate enhancer dysregulation as the basis by which cells acquire metastatic competence and demonstrate that such dysregulation presents an opportunity for the development of targeted anti-metastatic therapies, as illustrated at the $F 3$ locus.

\section{Methods}

\section{Cell Culture}

Human osteosarcoma cell lines were obtained and cultured as previously described ${ }^{21}$. MG63.3 cells were derived from MG63.2 (obtained from Dr. Hue Luu, University of Chicago, Chicago, IL) by metastatic selection in mice as previously described ${ }^{51}$.

The metastatic properties of these clonally related parental and metastatic cell lines have been thoroughly characterized in multiple murine models of metastasis ${ }^{21}$. A sample size of 5 cell line pairs was chosen to capture the spectrum of methods of metastatic derivation and to 
sufficiently power the study for comparative analyses based on similar studies completed by our lab in the past.

The purity and authenticity of all lines used in these studies has been independently confirmed by short tandem repeat (STR) profiling performed by the International Cell Line Authentication Committee. Mycoplasma testing was routinely performed with Mycoalert Mycoplasma detection kit (Lonza).

\section{Mouse Studies}

All animals were housed and handled in accordance with protocols approved by the CWRU IACUC or the NCI IACUC depending on location of performed studies. The number of animals included in each of the described studies was based on extensive past experience in the development and use of murine models of metastasis by our group. Each study was designed to minimize unnecessary animal use, optimize statistical power, and account for known variance in each model system. Within each experiment mice of the same strain, sex, and age were used for all conditions. At the initiation of each experiment mice were randomly assigned to cages and all mice in a given cage received equivalent treatment (e.g. doxycycline). Researchers were not blinded to the group assignments of mice as no subjective measurements were used.

\section{Human Subjects}

Osteosarcoma primary and lung metastatic tumors were obtained from the Laboratory of Experimental Oncology, Rizzoli Institute, Bologna, Italy with approval from Rizzoli Institute Ethics Committee. A waiver was granted for informed consent for patients deceased at the time of data collection according to the Data Privacy Regulation. Estimated tumor cellularity for samples ranged from $50-90 \%$.

\section{Ex Vivo Lung Metastasis Assay}

Procedure for RNA Isolation-GFP-positive tumor cells $\left(5 \times 10^{5}\right)$ were delivered by tail vein injection to 8-10 week old female SCID/Beige (Charles River). Within 15 minutes of tumor cell injection, mice were euthanized with $\mathrm{CO}_{2}$ inhalation, and lungs were insufflated with a mixed agarose/media solution. Lung sections for ex vivo culture were generated as described ${ }^{29}$ and incubated at $37^{\circ} \mathrm{C}$ in humidified conditions of $5 \% \mathrm{CO}_{2}$. Culture media was changed and lung sections were flipped every 2 days. Tumor cell RNA was harvested at $24 \mathrm{hr}$ and 14 day time points from one mouse for each condition. Lung sections were chopped into fine pieces and incubated in $3 \mathrm{ml} \mathrm{HBSS}$ with $1 \mathrm{mg} / \mathrm{ml}$ collagenase at $37^{\circ} \mathrm{C}$ for 30 minutes. EDTA was added to a final concentration of $10 \mathrm{mM}$ and the solution was placed on ice to stop digestion. Digested material was homogenized by passing through 18 ga needle $3-5 \mathrm{x}$ using $10 \mathrm{ml}$ syringe. Homogenate was passed through a 70 micron cell strainer (Corning Life Sciences) and centrifuged at $500 \mathrm{xg}$ for 5 minutes at $4^{\circ} \mathrm{C}$. Supernatant was aspirated and cells were re-suspended in 5ml ACK lysing buffer for 3 minutes at RT to lyse RBCs. Lysis was stopped by adding $10 \mathrm{ml}$ HBSS and cells were centrifuged at $500 \mathrm{xg}$ for 5 minutes at $4^{\circ} \mathrm{C}$. Supernatant was aspirated and cells were re-suspended in $2-3 \mathrm{ml} 0.5 \mathrm{mM}$ EDTA PBS and placed on ice. Immediately prior to sorting, cells were passed through a 40 micron cell strainer (Corning Life Sciences). 
Cells were sorted by FACS to isolate GFP+ tumor cells and immediately centrifuged at 500x $g$ for 5 minutes at $4^{\circ} \mathrm{C}$. Supernatant was aspirated and cells were lysed in $1 \mathrm{ml}$ TRIzol reagent (Life Technologies), RNA was extracted with 200ul chloroform. Organic phase was isolated and 700ul of EtOH added. RNA was purified from this solution using the RNeasy Micro Kit (Qiagen).

Procedure for F3 Knockdown Studies-F3 knockdown cells were pretreated with $5 \mathrm{ug} / \mathrm{ml}$ doxycycline for $40 \mathrm{hrs}$ in standard culture. F3 shRNA induced cells were sorted by FACS to isolate DsRed+/GFP+ fraction. Uninduced control cells were sorted to isolate GFP + fraction. $2 \times 10^{5}$ cells were injected into the tail vein of each mouse. For F3 knockdown condition $5 \mathrm{ug} / \mathrm{ml}$ doxycycline was added to agarose/media solution used to insufflate lungs as well as culture media. The medium was changed and fresh doxycycline was added every 2 days. A total of 8 lung sections were imaged for each condition (4 sections per mouse, 2 mice per condition).

Procedure for JQ1 Studies- $5 \times 10^{5}$ tumor cells were injected into the tail vein of each mouse. For JQ1 treated cultures, medium was supplemented to a final concentration of 250nM JQ1 by adding 10mM DMSO stock solution. Vehicle treated culture media was supplemented with DMSO volumes matching JQ1 treatment. Media was changed and fresh JQ1 or DMSO was added every 2 days. A total of 8 lung sections were imaged for each condition (4 sections per mouse, 2 mice per condition).

Assessment of Metastatic Burden-Lung sections were imaged by inverted fluorescent microscopy (Leica DM IRB) at a magnification of 2.5x. 2-3 images per lung section were taken to capture the entire surface of each section. Image analysis was performed using ImageJ software to quantify total GFP+ area per lung section. The metastatic burden was calculated by normalizing total GFP+ area to GFP+ area for each section on day 0 . Values reported represent mean normalized tumor burden for all sections for each condition (8 sections per condition).

\section{In Vitro RNA isolation}

To match conditions used to isolate cells from ex vivo lung sections, cells growing in vitro were trypsinized and exposed to the same mechanical/enzymatic digestion conditions and sorted by FACS as described above. $5 \times 10^{5} \mathrm{GFP}+$ cells were collected and RNA was isolated as described above.

\section{Code Availability}

All custom code used for analysis in the current study are available from the corresponding author on reasonable request.

\section{ChIP-seq}

ChIPs were performed from 5-10 $\times 10^{6}$ cross-linked cells and sequencing libraries were prepared as previously described ${ }^{52}$. The following antibodies were used for ChIP: rabbit anti-H3K4me1 (Abcam \#8895), rabbit anti-H3K27ac (Abcam \#4729), rabbit anti-c-Fos (Santa Cruz \#sc-52), rabbit anti-FOSL1/Fra-1 (Santa Cruz \#sc-605). ChIP-seq libraries were 
sequenced on the HiSeq 2000 or 2500 platform at the Case Western Reserve University Genomics Core Facility.

Analysis was performed as previously described ${ }^{8}$.

\section{Met-VEL Analysis}

H3K4me1 ChIP-seq peaks were filtered to remove all peaks overlapping ENCODE blacklisted regions for functional genomics analysis (https://sites.google.com/site/ anshulkundaje/projects/blacklists) as well as peaks $+/-1 \mathrm{~kb}$ from transcription start sites (TSSs) of all annotated RefSeq genes to exclude promoters. Resulting peak lists of parental and metastatic cell line pairs were merged and RPKM values within merged peaks were calculated. Gained and lost Met-VELs were called as peaks with 3-fold increased or decreased RPKM values in metastatic cell lines relative to parental cell lines, respectively. To determine the fraction of differentially active enhancers in different cell types (Extended Figure 2), H3K4me1 ChIP-seq peaks for each pair of samples were filtered for ENCODE blacklisted regions and promoters, concatenated, and merged. Peak RPKMs were calculated for each sample in a pair and floored to 0.3. Differentially active enhancers were defined as those showing a 3-fold change in $\mathrm{H} 3 \mathrm{~K} 4 \mathrm{me} 1$ signal in one sample relative to the other. The fractions of differentially active enhancers for the osteosarcoma tumors and cell lines panels were based on averages for each group.

\section{Met-VEL Clustering Analysis}

Global Met VEL distribution was assessed by calculating Met-VEL counts in 200kb sliding windows across all chromosomes. Met-VEL islands were defined as regions bordered by $200 \mathrm{~kb}$ windows with Met-VEL counts of $0.200 \mathrm{~kb}$ windows with maximum Met-VEL counts in each Met-VEL island were identified. To test for non-random Met-VEL distribution, the same analysis was performed on 1000 Met-VEL-size-matched H3K4me1 peak lists randomly sampled from all $\mathrm{H} 3 \mathrm{~K} 4 \mathrm{me} 1$ peaks in the cell line being analyzed to account for global enhancer distribution biases. Metastatic cell line H3K4me1 peaks were sampled to assess gained Met-VEL clustering. Parental cell line H3K4me1 peaks were sampled to assess lost Met-VEL clustering. The sampled lists were used to define expected distributions of random VEL acquisition in each cell line. Expected distributions were compared to observed distributions to test the null hypothesis of random Met-VEL acquisition. A p-value threshold of 0.05 was used to reject the null hypothesis in support of non-random acquisition of Met-VELs. 200kb windows with Met-VEL counts exceeding these thresholds were called as Met-VEL clusters.

\section{Super-Enhancers (SEs)}

Metastatic and parental cell line-specific SEs were identified from H3K27ac profiles using the ROSE software (retrieved from https://github.com/BradnerLab/pipeline). Analysis performed as previously described ${ }^{8}$.

\section{RNA-seq}

Gene expression profiles of cell lines grown in vitro were compared to expression profiles of the same cell lines at various time points during metastatic colonization using the ex vivo 
pulmonary metastasis assay. RNA quality was assessed by 2200 TapeStation Instrument (Agilent). PolyA+ RNA was isolated using the Illumina TruSeq RNA Sample Preparation Kit according to the manufacturer's protocol. RNA-seq libraries were sequenced on the Illumina HiSeq 2000 or 2500 platform at the Case Western Reserve University Next Generation Sequencing Core Facility.

Expression analysis was performed as previously described, aligning to the $\mathrm{hg} 19$ genome build $^{8}$. FPKMs were quantile normalized across all samples.

\section{Prediction of Gene Targets of Enhancers}

Enhancer-gene assignments were made as described in ${ }^{30}$. To identify Met-VEL gene targets in patient tissues for which we did not have RNA-seq data, we used the GREAT software package $^{59}$ to generate an initial list of candidate Met-VEL gene targets. We then further filtered this list by only including genes within the same TAD as the enhancer with a H3K27ac peak at their promoter, using a p-value cutoff of $\mathrm{P}<1 \mathrm{e}-5$. We then assessed overlaps in predicted Met-VEL gene targets between cell lines and patient tissues using the resulting gene lists.

\section{Gene Ontology Analysis}

Gained Met-VEL Gene Lists-Met-VEL gene lists were imported into gProfiler ${ }^{60}$ to generate enrichment scores for all GO, KEGG and REACTOME gene sets according to recommended settings for gProfiler http://baderlab.org/Software/EnrichmentMap/ GProfilerTutorial. Cytoscape (v3.2.1) and the Enrichment Map ${ }^{61}$ plug-in was used to generate networks for gene sets enriched with an FDR cutoff of $<0.05$.

Lost Met-VEL Gene Lists-For gene ontology (GO) analysis, the genes associated with Met-VELs were analyzed using DAVID (http://david.abcc.ncifcrf.gov/home.jsp). A p-value of $10^{-3}$ was used as the threshold for significant enrichment of an ontologic category. Categories significantly enriched for gained or lost Met VEL genes in 2 or more pairs are reported, limiting overlapping lists to the three top scoring categories in each cell line (i.e. the categories with the lowest p-values).

\section{DHS-seq}

6.4-56 $\times 10^{6}$ cells from each cell line were sequenced for DNase hypersensitivity (DHS) as previously described ${ }^{62}$. A $5^{\prime}$ phosphate added to linker 1B to increase ligation efficiency. After DNase concentrations were optimized for each line a total of approximately $1 \times 10^{6}$ cells from optimally digested conditions were processed for sequencing. Libraries were sequenced on the HiSeq 2500 platform at the Case Western Reserve University Genomics Core Facility. Analysis was performed as previously described ${ }^{8}$.

\section{Chromosome Conformation Capture Sequencing (4C-seq)}

$4 \mathrm{C}$-seq sample preparation was performed as previously described ${ }^{63}$. NlaIII served as a primary restriction enzyme, DpnII as a secondary 4 bp-cutter. Primer sequences are provided in below. Amplified sample libraries were pooled and spiked with $40 \%$ PhiX viral genome sequencing library to increase sample diversity. Multiplexed sequencing was performed on 
the MiSeq platform. Demultiplexing was performed by an in-house algorithm and all reads were hard trimmed to 36bp. Clipping of the primer sequences and data processing was performed using 4Cseqpipe Version 0.7 (retrieved from http:// compgenomics.weizmann.ac.il/tanay/?page_id=367). The viewpoint reads were aligned to a fragmented genome, as determined by the restriction site positions of the chosen primary and secondary restriction enzymes. A running linearly weighted mean, calculated in sliding windows of size 2-50KB, was used for signal smoothing of each genomic bin (size 16bp). Contact enrichment sites along the chromosomal axis were visually inspected.

\section{Motif Analysis}

To identify transcription factor (TF) motifs enriched in Met-VEL peaks, enhancers were centered on DNase hypersensitivity sites and the SeqPos module of the Cistrome tool was used to scan a $1 \mathrm{~kb}$ window for enriched curated motifs ${ }^{64}$. Significantly enriched motifs in each cell line were then filtered using RNA-seq data and only expressed TFs were used for downstream analysis. Expressed TFs with enriched motifs in 3 out of 3 metastatic/parental cell line pairs (MG63.3/MG63; MNNG/HOS; 143B/HOS) are presented in the results.

\section{In Vivo RNAi High-Throughput Functional Assay}

Vector Construction-The Tet-ON lentiviral construct was made by modifying the previously published optimized shRNAmir, "miR-E”, pRRL backbone ${ }^{36}$. Briefly, this construct contains an optimized $3^{\text {rd }}$ generation Tet-responsive element (T3G) and rtTA3 to potentiate a positive feedback loop, enhancing expression of the construct upon induction and reducing construct leakiness. The version of the construct that we modified contained a constitutive Venus reporter and an induced DsRed reporter of expression (LT3REVIR). The construct was modified using standard cloning techniques to replace the Venus reporter with a puromycin resistance element (renamed LT3REPIR) so that cells already constitutively expressing GFP could be selected for transduction.

shRNA Library Generation-shRNAs targeting 33 genes were selected from the transOMIC technologies shERWOOD-UltramiR shRNA library (3 to 4 shRNAs per gene). Cloning of shRNA into the backbone construct was performed on contract by transOMIC technologies. The following shRNA sequences included in the library are listed in Supplementary Table 3. Scores indicate shERWOOD metric of predicted potency of each shRNA as assigned by previously described algorithm ${ }^{65}$. NGS of \#N/A indicates that the shRNA failed to clone into the lentiviral backbone.

Lentiviral Production-VSV-G pseudotyped lentivirus was generated with standard laboratory techniques. Briefly, shRNA-LT3REPIR plasmids were co-transfected with packaging vectors psPAX2 and pCI-VSVG (Addgene) into 293FT cells using Cal-Phos Mammalian Transfection Kit (Clontech). Individual supernatants containing virus were

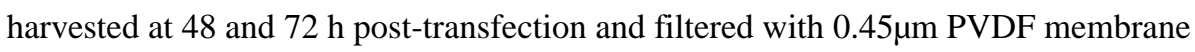
(Millipore).

Lentiviral Transduction and Selection-Transduction of MG63.3 was performed via $24 \mathrm{hr}$ exposure to lentivirus in the presence of $8 \mathrm{ug} / \mathrm{ml}$ polybrene using conditions to achieve 
$>1000 x$ coverage of each shRNA in the library. Infection rate estimated to be $0.135 \%$ and predicted to achieve predominantly one lentiviral integration per cell. Transduced and nontransduced cells were then treated with $2 \mathrm{ug} / \mathrm{ml}$ puromycin. Transduced cells were selected until all cells in non-transduced plate were dead (2-4days) to obtain a pure population of transduced cells (MG63.3i).

High-Throughput In Vivo Functional Assay-8-10 week old female SCID/Beige (Charles River) mice were used for the in vivo study arm. Mice were fed Dox Diet pellets containing 200mg/kg doxycycline (Bio-Serve) for 5 days prior to injection of cells. MG63.3i cells were pre-treated with $5 \mathrm{ug} / \mathrm{ml}$ doxycycline for $12 \mathrm{hrs}$ in standard culture before being delivered to mouse lungs by tail vein injection. This primes the cells, but knockdown is not achieved even at the transcript level until $24-72 \mathrm{~h}$ after doxycycline addition. $1.5 \times 10^{6}$ cells were injected into each mouse $(n=15)$. Mice were maintained on Dox Diet throughout the 21-day course of the experiment. At the conclusion of the experiment mice were euthanized by $\mathrm{CO}_{2}$ inhalation and lungs were surgically extracted and homogenized using the Tumor Dissociation Kit, human (Miltenyi) according to the manufacturer's protocol. Mouse lung cells were depleted using the Mouse Cell Depletion Kit (Miltenyi) according to the manufacturer's protocol. Lungs from 5 mice were pooled for each replicate to achieve 1000x engraftment coverage of each shRNA in the library. GFP+/DsRed+ cells were then isolated by FACS.

Three replicates of $1.5 \times 10^{6} \mathrm{MG} 63.3 \mathrm{i}$ cells growing in vitro were induced with $5 \mathrm{ug} / \mathrm{ml}$ doxycycline and maintained on doxycycline over 21 days in culture. GFP+/DsRed+ cells were isolated by FACS. Sorted cell counts of in vitro replicates were matched to numbers of cells isolated from the in vivo arm $\left(3-5 \times 10^{5}\right)$.

DNA was isolated from three replicates of uninduced MG63.3i cells as well as in vivo and in vitro arms of the experiment.

shRNA Amplification and Sequencing-Genomic DNA was isolated and sequenced as described ${ }^{66}$ with slight modification. Genomic DNA was isolated by two rounds of phenol extraction using PhaseLock tubes (5prime) followed by isopropanol precipitation. Deep sequencing libraries were generated by PCR amplification of shRNA guide strands using barcoded primers that tag the product with standard Illumina adapters. For each sample, DNA from at least $3 \times 10^{5}$ cells was used as template in multiple parallel 50- $\mu \mathrm{l} \mathrm{PCR}$ reactions, each containing $1 \mu \mathrm{g}$ template, $1 \times$ AmpliTaq Gold buffer, $0.2 \mathrm{mM}$ of each dNTP, $0.3 \mu \mathrm{M}$ of each primer and 2.5 U AmpliTaq Gold (Applied Biosystems), which were run using the following cycling parameters: $95^{\circ} \mathrm{C}$ for $10 \mathrm{~min} ; 35$ cycles of $95^{\circ} \mathrm{C}$ for $30 \mathrm{~s}, 52^{\circ} \mathrm{C}$ for $45 \mathrm{~s}$ and $72{ }^{\circ} \mathrm{C}$ for $60 \mathrm{~s} ; 72{ }^{\circ} \mathrm{C}$ for $7 \mathrm{~min}$. PCR products (340 nt) were combined for each sample, precipitated and purified on a $2 \%$ agarose gel (QIAquick gel extraction kit, Qiagen). Libraries were sequenced on the HiSeq 2500 platform at the Case Western Reserve University Genomics Core Facility. Libraries were sequenced using a primer that reads in reverse into the guide strand (miR30EcoRISeq, TAGCCCCTTGAATTCCGAGGCAGTAGGCA). To provide a sufficient baseline for detecting shRNA depletion in experimental samples, we aimed to acquire $>1,000$ reads per shRNA in all samples or $1.37 \times 10^{5}$ reads per sample. In practice, we achieved $>2 \times 10^{6}$ reads 
for all samples. Sequence processing was performed using two custom workflows using usegalaxy.org ${ }^{67}$. Workflow can be accessed by the following links: https://usegalaxy.org/u/ tyleremiller/w/shrna-pipeline1 and https://usegalaxy.org/u/tyleremiller/w/shrnastep2. For each shRNA and condition, the number of matching reads was normalized to the total read number per lane. This measure of normalized coverage was used for all downstream analyses.

\section{Inducible Knockdown of Tissue Factor (F3)}

shRNAs targeting tissue factor (F3) were selected from the transOMIC technologies shERWOOD-UltramiR shRNA library and cloned into the LT3REPIR as described in the preceding section. Cloning of shRNA into the backbone construct was performed on contract by transOMIC technolgies. The following shRNA sequences were tested:

\section{Table 2-3}

Hairpins used in $F 3$ knockdown experiments

\begin{tabular}{|c|c|c|c|}
\hline Gene & Hairpin & ID & Sequence \\
\hline F3 & shF3A & RLGH-DU16277 & TGCTGTTGACAGTGAGCGATCAGAAGGAACAACACTTTCATAGTGAAGCCACAGATGTATGAAAG \\
\hline F3 & shF3B & RLGH-DU14137 & TGCTGTTGACAGTGAGCGCGCCAACAATTCAGAGTTTTGATAGTGAAGCCACAGATGTATCAAAAC \\
\hline F3 & shF3C & RLGH-GU22081 & TGCTGTTGACAGTGAGCGCCCGACGAGATTGTGAAGGATATAGTGAAGCCACAGATGTATATCCTT \\
\hline
\end{tabular}

Lentiviral Production-VSV-G pseudotyped lentivirus was generated with standard laboratory techniques. Briefly, shRNA-LT3REPIR plasmids were cotransfected with packaging vectors psPAX2 and pCI-VSVG (Addgene) into 293FT cells using Cal-Phos Mammalian Transfection Kit (Clontech). Individual supernatants containing virus were harvested at 48 and $72 \mathrm{~h}$ post-transfection and filtered with $0.45 \mu \mathrm{m}$ PVDF membrane (Millipore).

Lentiviral Transduction and Selection-Transduction was performed via $24 \mathrm{hr}$ exposure to lentivirus in the presence of $8 \mathrm{ug} / \mathrm{ml}$ polybrene. Transduced and non-transduced cells were then treated with $2 \mathrm{ug} / \mathrm{ml}$ puromycin. Transduced cells were selected until all cells in non-transduced plate were dead (2-4days).

Assessment of Knockdown-Optimal shRNA induction was assessed and found to occur with $5 \mathrm{ug} / \mathrm{ml}$ doxycycline treatment. MG63.3 cells transduced with shF3A, shF3B, and shF3C were treated with doxycycline for $40 \mathrm{hrs}$, typsinized and sorted to isolate DsRed $+/ \mathrm{GFP}+$ fraction. Uninduced cells were sorted for GFP+ fraction. RNA was extracted from $1 \times 10^{6}$ cells and purified using the RNAeasy Micro kit (Qiagen) according to the manufacturer's protocol. RNA quality was assessed by 2200 TapeStation Instrument (Agilent). cDNA was synthesized using the High Capacity RNA-to-cDNA kit (ABI) according to the manufacturer's protocol. Knockdown efficiency was determined by RTqPCR for $F 3$ using optimized TaqMan Gene Expression Assay primers and TaqMan Gene Expression Master Mix (Life Technologies). 
RT-qPCR was performed to quantify percent knockdown with induction of each hairpin relative to uninduced controls. Hairpins shF3A and shF3B showed the highest degree of F3 knockdown and were chosen for use the remainder of these studies.

\section{In Vivo Experimental Metastasis Model}

8-10 week old female SCID/Beige (Charles River) mice were used for all experimental metastasis studies. For all in vivo experimental $F 3$ knockdown studies, mice in the $F 3$ knockdown group were fed Dox Diet pellets containing $2 \mathrm{gm} / \mathrm{kg}$ doxycycline (Bio-Serve) for 5 days prior to injection of cells. $F 3$ knockdown cells were pre-treated with $5 \mathrm{ug} / \mathrm{ml}$ doxycycline for $24 \mathrm{hrs}$ in standard culture. F3 shRNA induced cells were sorted by FACS to isolate DsRed+/GFP+ fraction. Control cells were sorted to isolate GFP+ fraction.

End Point Assessment of Lung Metastasis- $5 \times 10^{5}$ MG63.3 DsRed+/GFP+ or MG63.3 GFP+ cells were injected into the tail vein of each mouse ( $n=10$ mice per condition). Mice in the F3 knockdown group were maintained on Dox Diet throughout the experiment. On day 7 or day 14 following injection 5 mice from each group were euthanized by $\mathrm{CO}_{2}$ inhalation. Lungs were insufflated with PBS and imaged by inverted fluorescent microscopy (Leica DM IRB) at a magnification of 2.5x. 5 images per lung were taken to assess metastatic burden in each mouse.

Image analysis was performed using ImageJ software to quantify total GFP+ area per image. The metastatic burden was calculated as the sum of the total GFP+ area in the 5 images from each mouse.

Survival Analysis $-5 \times 10^{4}$ cells were injected into the tail vein of each mouse ( $\mathrm{n}=5$ mice per condition). Mice in the F3 knockdown group were maintained on Dox Diet throughout the experiment. All mice that died underwent complete necropsy examination and confirmation of metastasis.

\section{Orthotopic Spontaneous Lung Metastasis Model}

8-10 week old female NSG mice (Jackson) were used for spontaneous metastasis studies. Mice in the F3 knockdown group were given water supplemented with $2 \mathrm{mg} / \mathrm{ml}$ doxycycline hyclate (Sigma) and 2\% sucrose for 5 days prior to injection of cells. F3 knockdown cells were pre-treated with $5 \mathrm{ug} / \mathrm{ml}$ doxycycline for $24 \mathrm{hrs}$ in standard culture. F3 shRNA induced cells were sorted by FACS to isolate DsRed+/GFP+ fraction. Control cells were sorted to isolate GFP+ fraction. $3 \times 10^{5}$ cells were injected orthotopically into the paraosseous region adjacent to the left proximal tibia. For the F3 knockdown group water was changed and fresh doxycycline was added twice weekly. Injection sites were monitored twice weekly for tumor formation.

Tumors became measureable on day 21 for all groups at which time tumors were measured in two dimensions twice weekly. Tumor volume was calculated as follows: volume $\left(\mathrm{mm}^{3}\right)=$ $3.14 \times[$ long dimension $(\mathrm{mm})] \times[\text { short dimension }(\mathrm{mm})]^{2}$. Experiment was terminated following 21 days of tumor measurements (42 days after injections) and mice were euthanized by $\mathrm{CO}_{2}$ inhalation. Lungs were insufflated with PBS and imaged by fluorescent 
microscopy at 2.5x using Leica DM 5500B light microscope with a Leica DFC 500 camera. 5 images per lung were taken to assess metastatic burden in each mouse.

Image analysis was performed using ImageJ software. Micrometastases were defined as GFP+ lesions with diameter $>25$ pixels in images captured at $2.5 \mathrm{x}$ magnification. Number of micrometastases per image were manually counted. Total micrometastases for each lung were calculated as the sum of the total number of micrometastases in 5 images from each mouse.

\section{In Vitro Assay for FXa Formation}

Cells growing in vitro were pre-treated with $25 \mu \mathrm{g} / \mathrm{mL}$ IgG control, Mab-10H10, or Mab-5G9 20 minutes prior to assay. Cells were washed in serum-free DMEM, and Xa generation over time was measured 30 minutes after the addition of 1nM FVIIa and 50nM FX using the chromogenic substrate Spectrozyme FXa.

\section{Assessment of F3 Inhibiting Antibodies on Metastatic Progression}

$5 \times 10^{5}$ GFP+ MG63.3 cells were mixed with 500 $\mu$ IgG control, Mab-10H10, or Mab-5G9 and injected into the tail vein of 10-12 week old female SCID-beige mice ( $>5$ mice per group). Mice were sacrificed 14 days after injection and metastatic burden was assessed by whole lung fluorescent imaging (5 images per mouse). Metastatic burden was quantified as total GFP+ area per mouse.

\section{F3 Lung Metastasis Staining}

To assess the $\mathrm{F} 3$ expression in metastatic tumor cells in the lung at progressive time points, mice were injected with $1 \times 10^{6}$ cells (via tail vein) and were euthanized via $\mathrm{CO}_{2}$ inhalation at $24 \mathrm{hrs}$ and 15 days post-injection. Lungs were harvested, formalin-fixed and paraffin embedded. For ex vivo lung metastasis staining, the protocol described above was followed and lung sections were fixed at $24 \mathrm{hrs}$ and 5 days post-injection. Tissue sections of lungs were cut at a thickness of 5 microns. Prior to immunostaining, paraffin sections were dewaxed with xylenes, and rehydrated with an ethanol series. For antigen retrieval, tissue sections were immersed $95^{\circ} \mathrm{C}$ Target Retreival Solution (DAKO) for 25 minutes. Tissue sections were permeabilized with $0.01 \%$ Triton- $X$ in PBS for 10 minutes. Slides were rinsed with PBS and blocked with 4\% BSA in PBS for 10 minutes. The following primary antibodies were used: F3 - Rabbit monoclonal IgG F3 antibody (ab151748, Abcam); GFP Goat polyclonal IgG GFP antibody conjugated to FITC (ab6662, Abcam). Primary antibodies were diluted in 4\% BSA 1:100 and slides were incubated in antibody solution at $4^{\circ} \mathrm{C}$ overnight. Slides were rinsed and incubated with goat polyclonal $\mathrm{IgG}$ anti-rabbit $\operatorname{IgG}(\mathrm{H}$ $+\mathrm{L}$ ) conjugated to Alexa 594 (A-11037, Life Technologies) diluted 1:200 in 4\% BSA for 1hr in dark humidified slide chamber. Nuclei were visualized with DAPI (Sigma, 1ug/ml). Tissue sections were mounted on slides using anti-fade mounting medium (Vectashield).

Stained sections were imaged by fluorescent microscopy at 20 or 40x using Leica DM 5500B light microscope with a Leica DFC 500 camera. Image analysis was performed using ImageJ software. F3 expression was computed within GFP+ metastatic tumor cell area. 


\section{Tissue Array Staining and Scoring}

A tissue microarray that we previously developed containing 20 osteosarcoma patient lung metastases ${ }^{68}$ was assessed for $\mathrm{F} 3$ expression. 18 of these 20 samples had cores of sufficient quality on the stained slide to be scored. For staining, paraffin was removed by 5 minute incubation in xylene bath $\mathrm{x} 2$ and rehydrated using step-down concentrations of EtOH. Antigen retrieval was performed by incubation in 1:10 dilution of Target Retrieval Solution (DAKO) in steamer for 25 minutes at $95^{\circ} \mathrm{C}$. Cells were permeabilized with $0.01 \%$ Triton-X in PBS for 10 minutes. F3 immunohistochemical staining was performed using rabbit monoclonal IgG F3 antibody (ab151748, Abcam) and the EnVision+ System-HRP (Dako) according to the manufacturer's protocol. Cover slips were mounted on slides using antifade mounting medium.

The array was scored by the Director of Soft Tissue Pathology at the Cleveland Clinic and Learner Research Institute who was blinded to the sample type. Cores were scored based on the $\%$ of tumor cells in the core with positive staining for $\mathrm{F} 3(0=0 \%, 1+=1-25 \%, 2+=26-$ $50 \%, 3+=>50 \%$ ) and the intensity of $\mathrm{F} 3$ staining in positive areas (low intensity staining, high intensity staining). Individual cores were excluded from the analysis if no tumor was present, tumor was predominantly necrotic, or core was falling off the slide.

\section{Targeted Deletion of an F3 Met-VEL by Genome Engineering}

Two TALEN dimers were designed to target the flanks of the Met-VEL in the F3 locus as indicated in Fig. 5a. TALEN dimers recognized the sequences $5^{\prime}-$ GACCAACTCACTTGAGCTGtgtggtttttttCAGTGCACAATTGTGAAAT-3' and 5' GAATCGACTGATCAAAGCacatgaacttttaaaaaaGAGTAATAAGTTTACTT-3', where spacer elements are in lower case. TALEN constructs were assembled with adaptations of previously described protocols ${ }^{69,70}$. MG63.3 cells were grown in 6-well plate format to $70 \%$ confluence and transfected with $2.5 \mu \mathrm{g}$ plasmid for each TALEN monomer using Lipofectamine 2000® (ThermoFisher Scientific) as per the manufacturer's instructions. Cells were incubated for $48 \mathrm{~h}$ at $30^{\circ} \mathrm{C}$ and genomic DNA was subsequently harvested using QuickExtract ${ }^{\mathrm{TM}}$ DNA Extraction Solution (Epicentre) as recommended by the supplier. Efficient deletion of the F3 Met-VEL was confirmed by agarose gel electrophoresis of PCR products generated using primers $5^{\prime}$-GCAGTGCACAACCTGTACAAC- $3^{\prime}$ and $5^{\prime}$ TTGGCCAGGGTCATTATGTT-3' (Integrated DNA Technologies) and high fidelity AccuPrime $^{\mathrm{TM}}$ Taq DNA Polymerase (ThermoFisher Scientific). Single cell clones were derived by limiting dilution and genotypes were confirmed as described above. Enhancer deletion of clonal cell population used for functional metastasis experiments was confirmed by Sanger sequencing using the primer sequences listed above. 


\section{Extended Data}
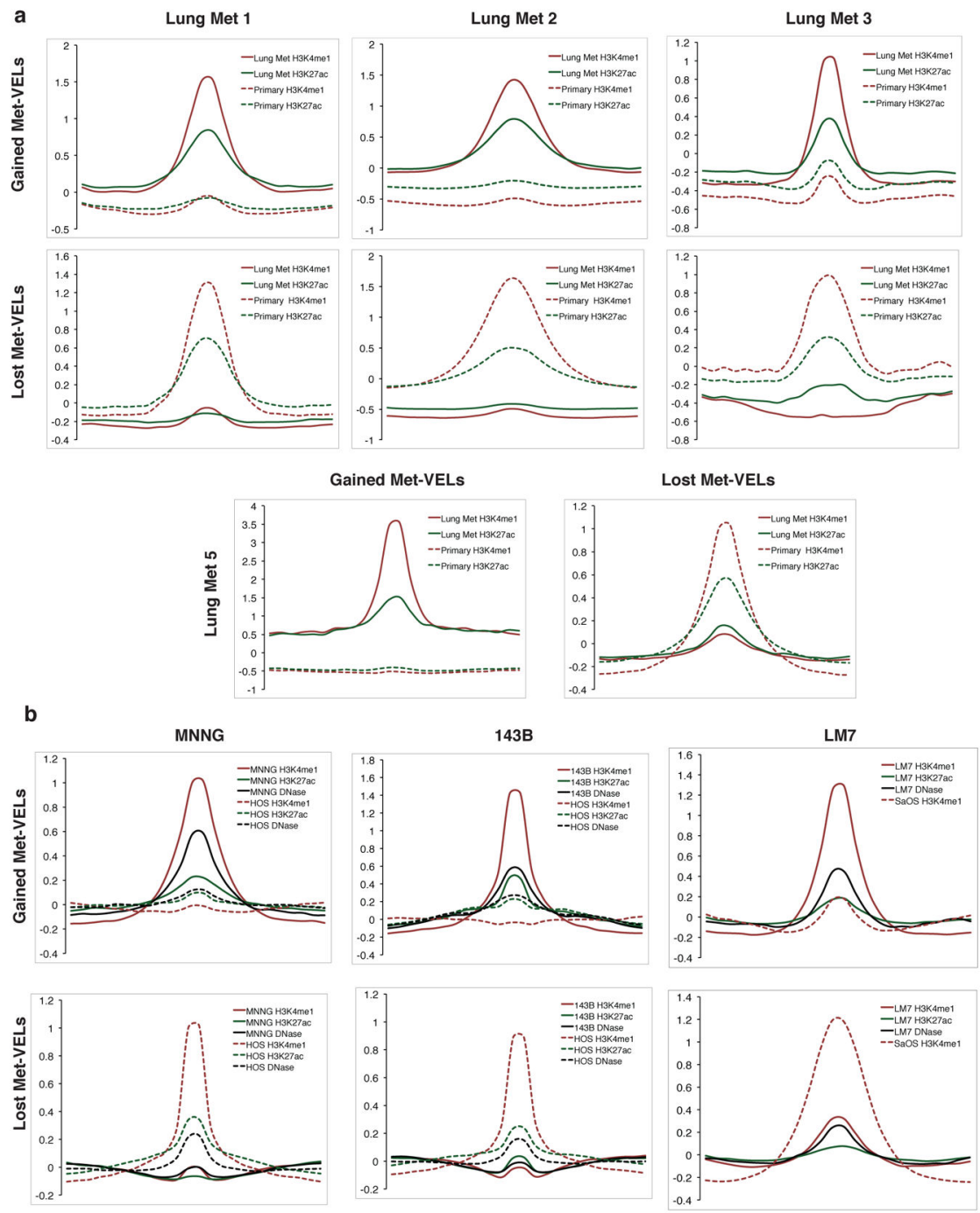

Gained Met-VELs
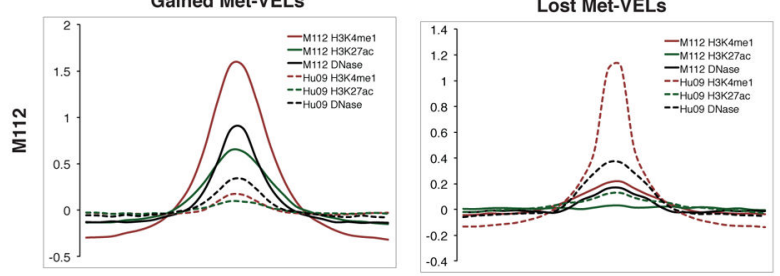

Extended Data Figure 1. Met-VEL profiles of osteosarcoma patient lung metastases and human osteosarcoma cell lines

a, Aggregate plots showing H3K4me1 ChIP-seq and H3K27ac ChIP-seq signal +/- 3Kb from midpoints of gained and lost Met-VELs in paired patient lung metastases and primary tumors.

b, Aggregate plots showing H3K4me1 ChIP-seq, H3K27ac ChIP-seq and DNase-seq signal $+/-3 \mathrm{~Kb}$ from mid-points of gained and lost Met-VELs in metastatic/parental human osteosarcoma cell lines. 


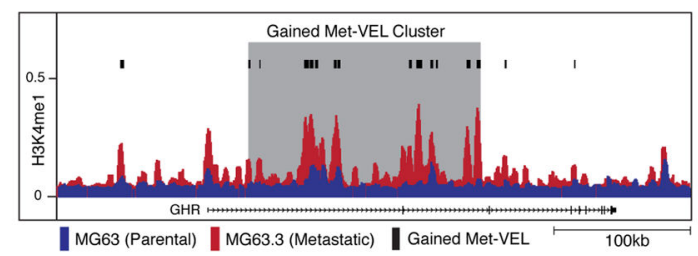

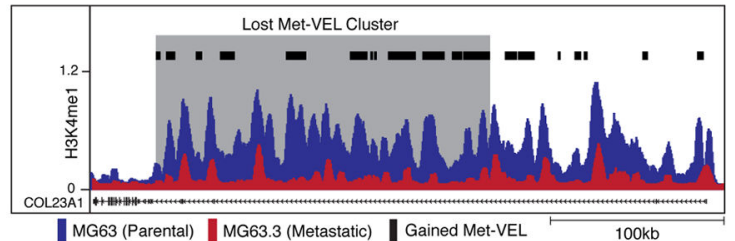

MG63 (Parental) MG63.3 (Metastatic) Gained Met-VEL b

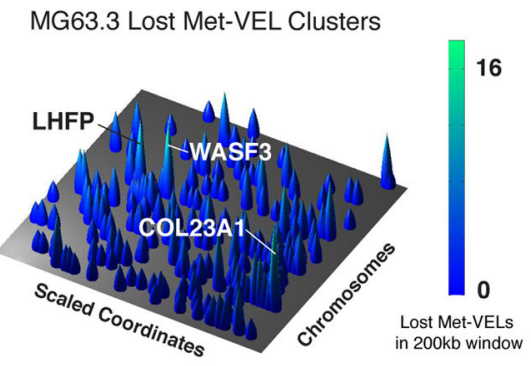

c d Gained Met-VELs

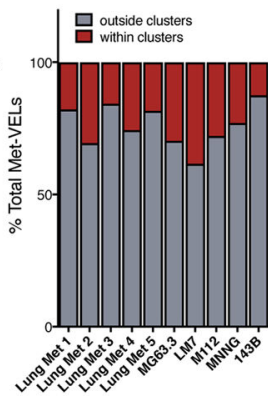

Lost Met-VELs

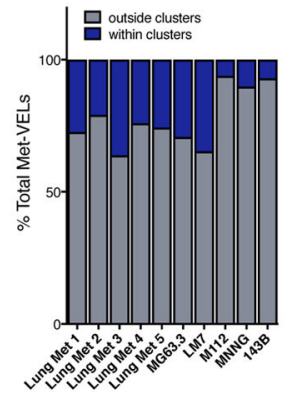

Extended Data Figure 2. Met-VEL clusters occur across metastatic cancers a. UCSC browser view of H3K4me1 profiles in MG63.3 (metastatic) and MG63 (parental) cell lines illustrating an example of a gained (left) and lost (right) Met-VEL cluster. MetVELs identified by black bars. 200kb Met-VEL clusters highlighted in gray.

b. Genome-wide lost Met-VEL landscape for MG63.3 cell line. Rows represent scaled chromosomal coordinates. Peaks represent maximum gained Met-VEL counts in $200 \mathrm{~kb}$ sliding windows. Predicted target genes for selected peaks are labeled.

c. Gained and lost Met-VEL cluster counts in patient lung metastases/primary tumors and metastatic/parental cell line pairs.

d. Percentage of total gained (top) and lost (bottom) Met-VELs within and outside of clusters in patient lung metastases/primary tumors and metastatic/parental cell line pairs. 


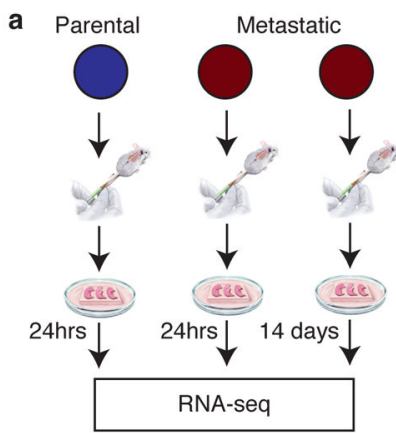

d

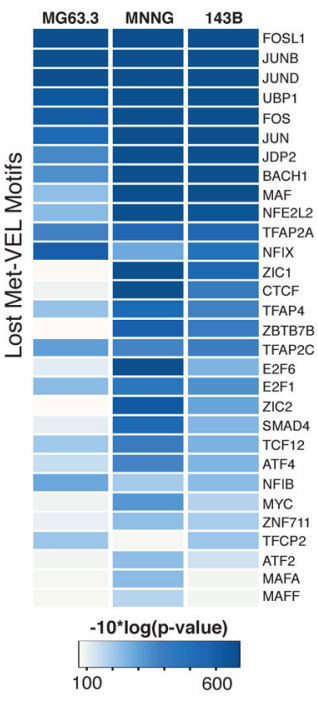

Lost Met-VEL Genes

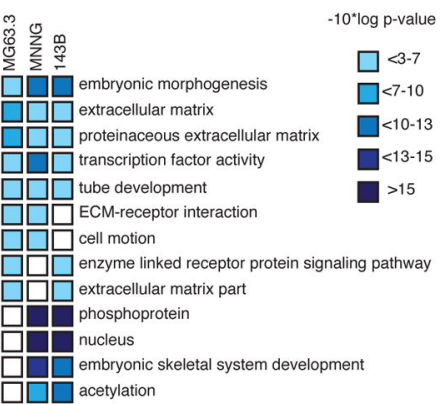

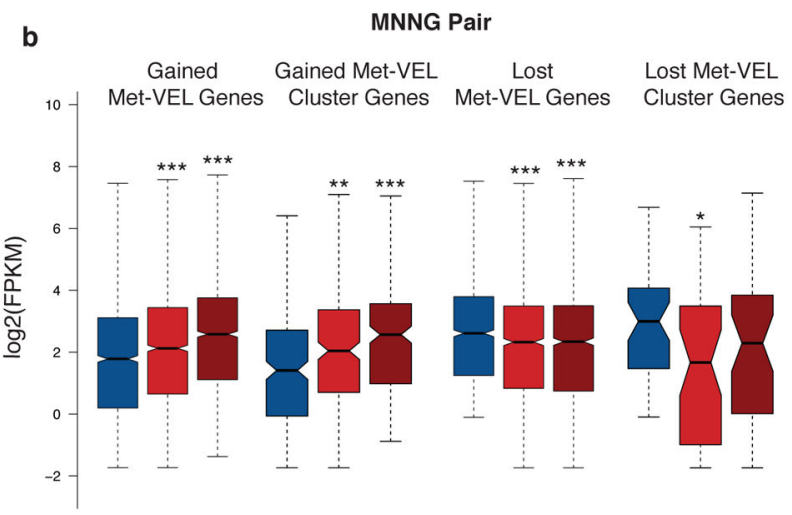

143B Pair

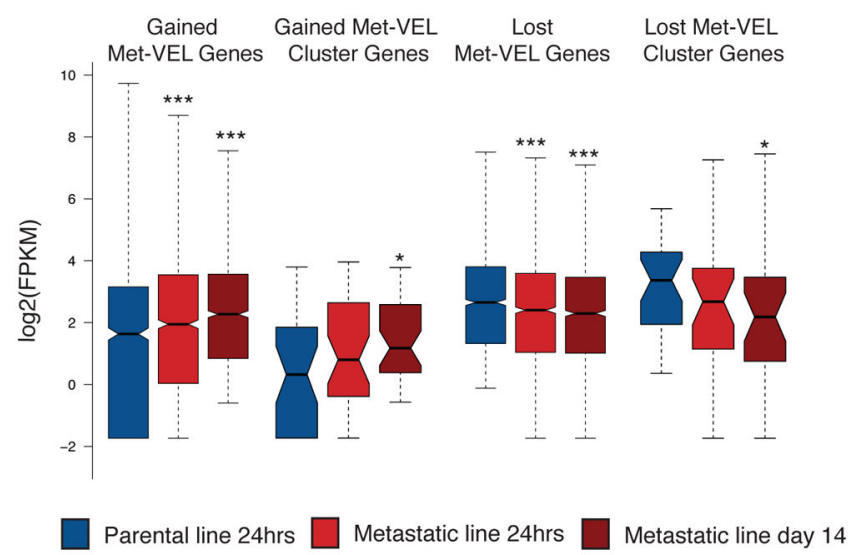

C

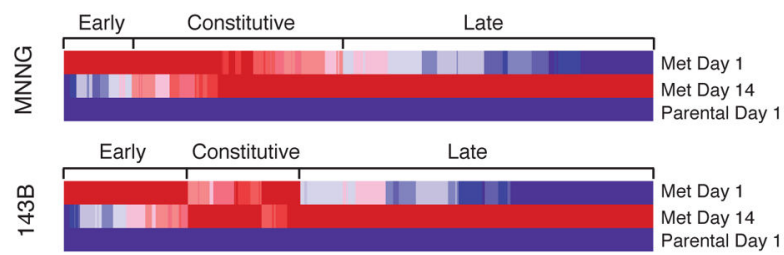

Normalized Expression

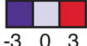

Extended Data Figure 3. Assessment of Met-VEL associated gene expression during metastatic colonization of the lung

a. Schematic of experimental design for assessment of Met-VEL gene expression in parental and metastatic cell lines in ex vivo lung metastasis model. Image adapted from ${ }^{29}$.

b. Log2 quantile-normalized FPKM values for gained (left) and lost (right) Met-VEL and Met-VEL cluster genes in HOS/MNNG (top) and HOS/143B (bottom) cell line pairs. Asterisks indicate significant differences in FPKM distributions between parental and metastatic cell lines (* $\mathrm{P}<0.05$; ** $\mathrm{P}<1 \mathrm{E}-3$; *** $\mathrm{P}<1 \mathrm{E}-4)$. P-values calculated by MannWhitney Test. 
c. Heatmap of up-regulated gained Met-VEL genes in HOS/MNNG (top) and HOS/143B (bottom) cell line pairs.

d. Expressed transcription factors with enriched motifs in lost Met-VELs in three metastatic/ parental cell line pairs and corresponding motif enrichment p-values.

e. Gene Ontology (GO) terms for lost Met-VEL genes in three metastatic/parental cell line pairs and corresponding $\mathrm{p}$-values.
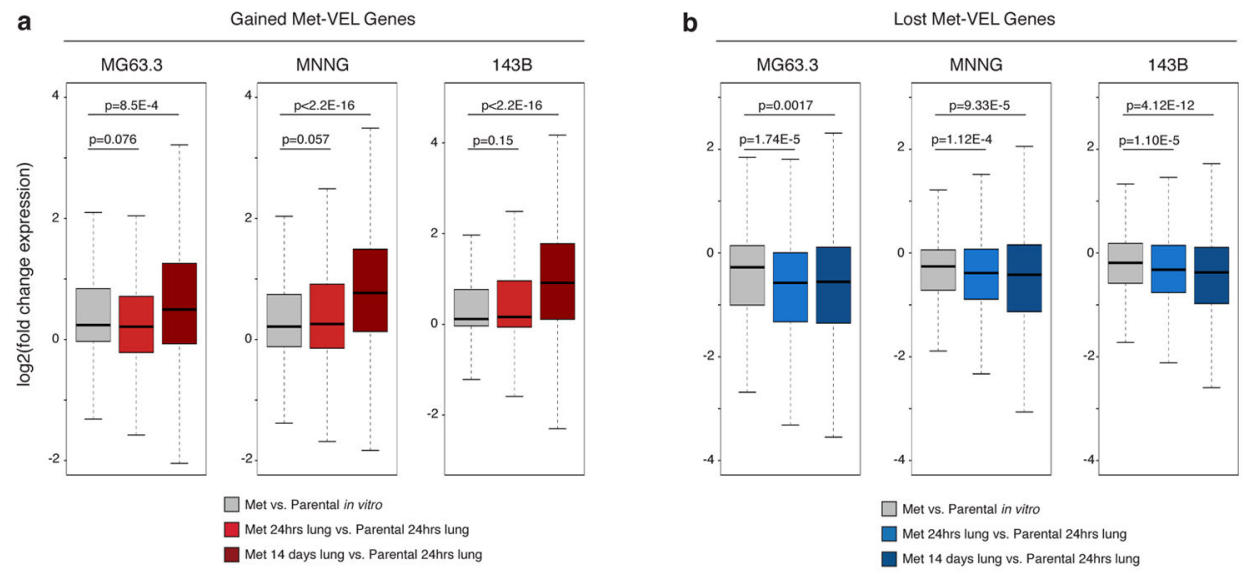

\section{C}
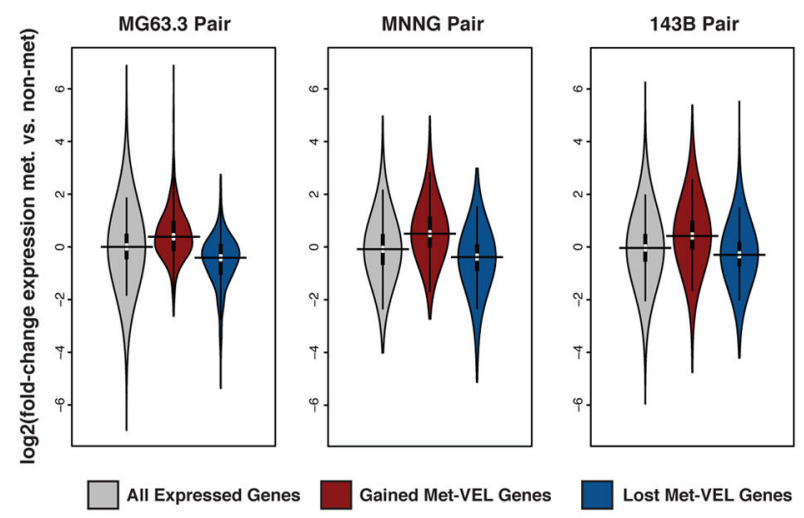

d
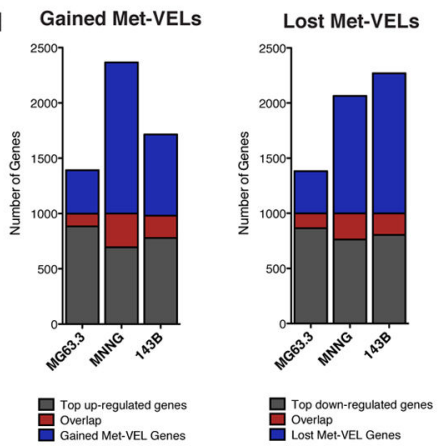

Extended Data Figure 4. Assessment of Met-VEL associated gene expression in vitro and in $e x$ vivo lung culture 
a. Log2 fold-change in quantile-normalized FPKM values of gained Met-VEL genes in metastatic cell lines versus parental cell lines in various conditions. P-values calculated by Mann-Whitney Test.

b. Log2 fold-change in quantile-normalized FPKM values of lost Met-VEL genes in metastatic cell lines versus parental cell lines in various conditions. P-values calculated by Mann-Whitney Test.

c. Violin plots of $\log 2$ (fold-change quantile-normalized FPKM values) distributions for gene sets in three metastatic cell lines relative to parental non-metastatic lines. Fold-change values for all expressed genes and gained Met-VEL gene sets represent comparisons of expression in metastatic lines at day 14 in ex vivo lung culture to non-metastatic lines at $24 \mathrm{hrs}$. Foldchange values for lost Met-VEL gene set represent comparison of expression in metastatic lines at $24 \mathrm{hrs}$ in ex vivo lung culture to non-metastatic lines at 24hrs.

d. Bar charts indicating overlap between gained (left) and lost (right) Met-VEL gene sets and top 1000 up- and down-regulated genes in corresponding conditions, respectively.

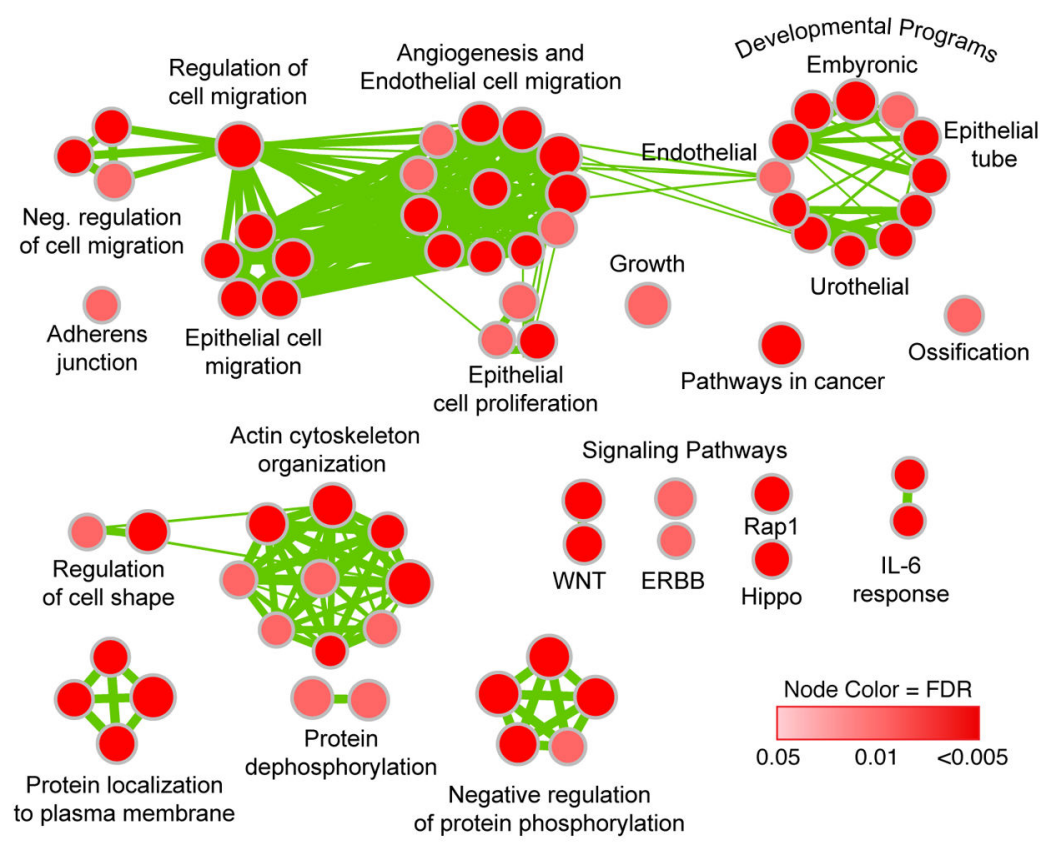

Extended Data Figure 5. Assessment of enriched functions of gained Met-VEL genes in osteosarcoma patient metastases

Enriched Map representation of all Gene Ontology (GO) terms for gained Met-VEL genes calculated by aggregating gene lists from two patient metastases (Lung Met 4 and Lung Met $5)$. 

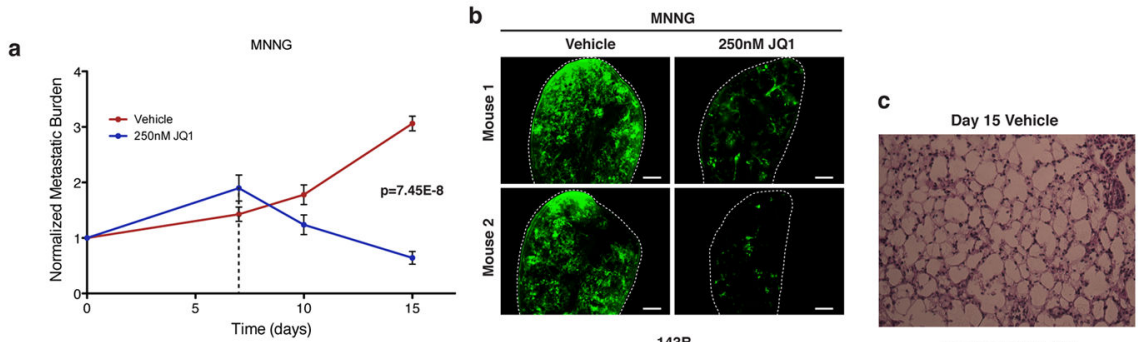

1438
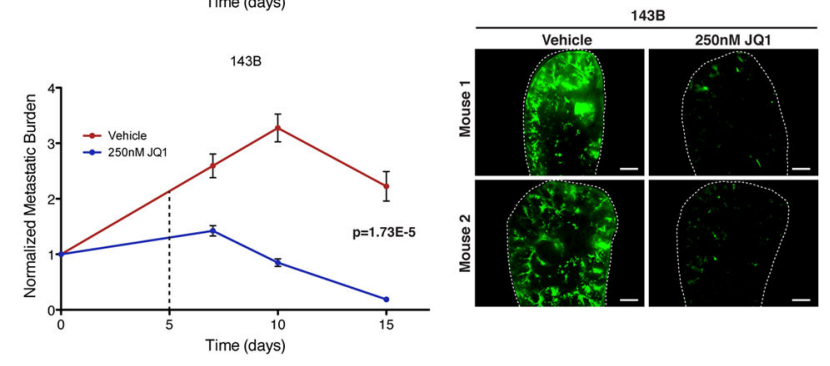

Day 15 250nM JQ1

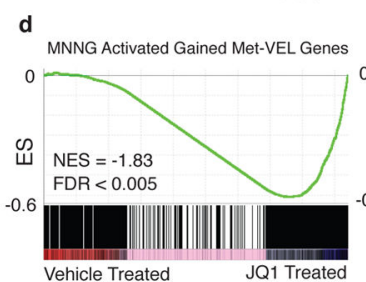

143B Activated Gained Met-VEL Genes

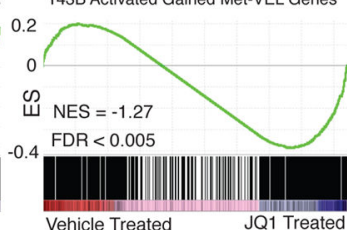

e
MNNG Activated Met-VEL Gene Expression

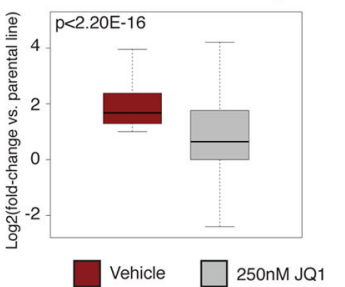

Vehicle Treated JQ1 Treated

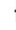

143B Activated Gained Met-VEL Genes

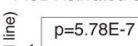
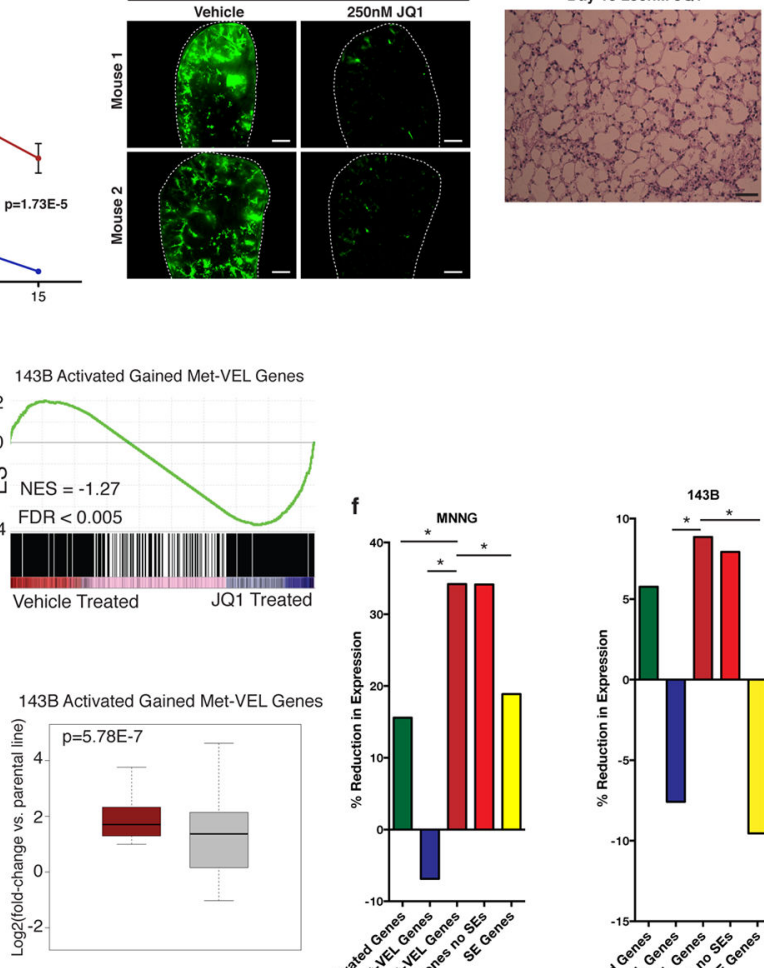

Extended Data Figure 6. Analysis of anti-metastatic and gene expression effects of BET inhibition with JQ1

a. Kinetics of metastatic outgrowth of MNNG (top) and 143B (bottom) metastatic cell lines in ex vivo lung culture with 250nM JQ1 or vehicle (DMSO) treatment. Metastatic burden measured as total GFP+ area per lung section normalized to GFP+ area on day 0. Values represent averages of 8 lung sections (4 sections per mouse x 2 mice) +/-SEM. P-value calculated by Mann-Whitney Test. Dashed line indicates time points chosen for RNA-seq studies.

b. Representative 2.5x images of vehicle (left) and 250nM JQ1 treated (right) lung sections at day 15. Lung sections outlined with dashed white line. Scale bar $=500 \mu \mathrm{m}$.

c. $20 \mathrm{X}$ image of hematoxylin and eosin stained section of lung slice after 15 days in ex vivo culture treated with DMSO (top) and 250nM JQ1 (bottom) illustrating viable lung cells and architecture. Scale bar $=100 \mu \mathrm{m}$. 
d. GSEA plots of 2-fold up-regulated gained Met-VEL gene sets in vehicle versus JQ1treated MNNG (left) and 143B (right) cells isolated from ex vivo lung culture. Cells isolated at time points indicated by dashed lines in Supplemental Figure 2-5a.

e. $\log 2$ fold-change expression 2-fold up-regulated gained Met-VEL gene sets in vehicle versus JQ1-treated MNNG (left) and 143B (right) cells isolated from ex vivo lung culture at time points indicated by dashed lines in Extended Data Figure 7a relative to parental cell line.

f. Percent reduction in gene expression with $250 \mathrm{nM} \mathrm{JQ1} \mathrm{treatment} \mathrm{of} \mathrm{all} \mathrm{genes} \mathrm{up-regulated}$ 2-fold in metastatic cell lines relative to parental cell lines at sorting time points, lost MetVEL genes, gained Met-VEL genes 2-fold up-regulated relative to parental cell lines at sorting time points, up-regulated gained Met-VEL genes without SE genes, and all SE genes in MNNG and 143B cells growing in ex vivo lung culture sorted at time points indicated in Extended Data Figure 7a.

a

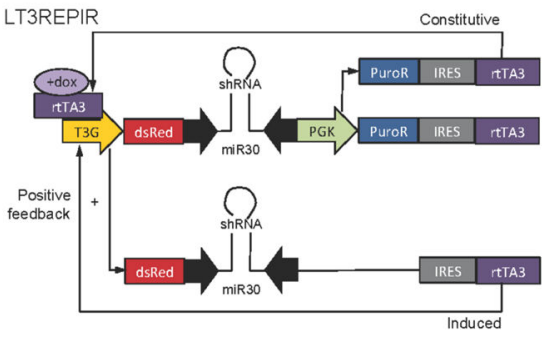

b

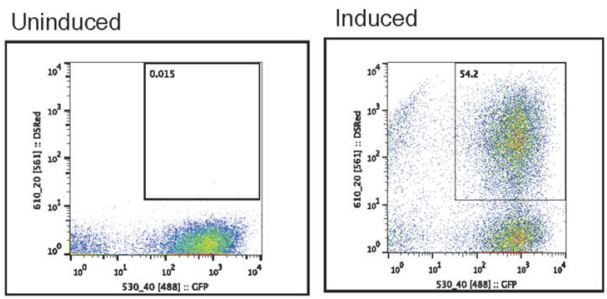

Extended Data Figure 7. Assessment of LT3REPIR shRNA construct induction and leakiness a. Schematic of doxycycline-inducible LT3REPIR shRNA construct. Modified from (Fellmann et al., 2013).

b. Cytometric analysis of GFP and DsRed expression in GFP+ MG63.3 cells transduced with LT3REPIR construct at baseline (left) and 40hrs after exposure to $5 \mathrm{ug} / \mathrm{ml}$ doxycycline (right). 
a

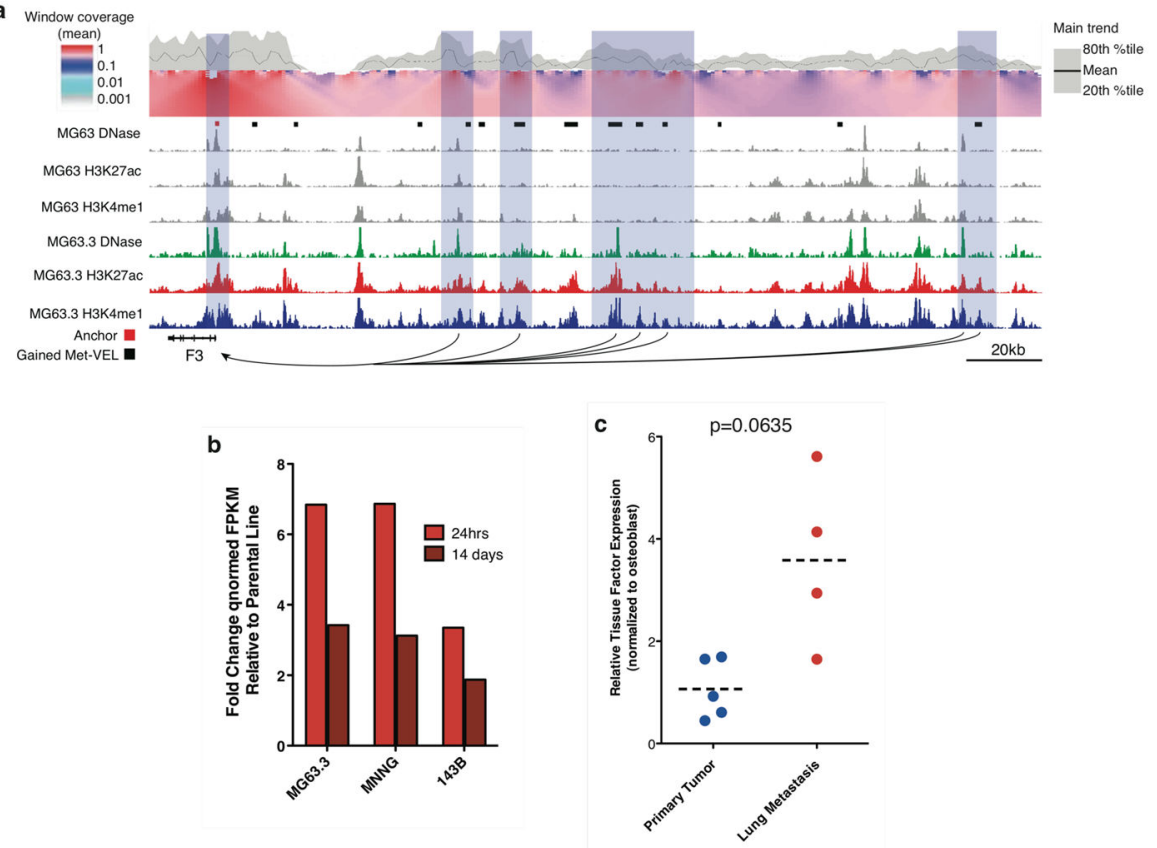

d

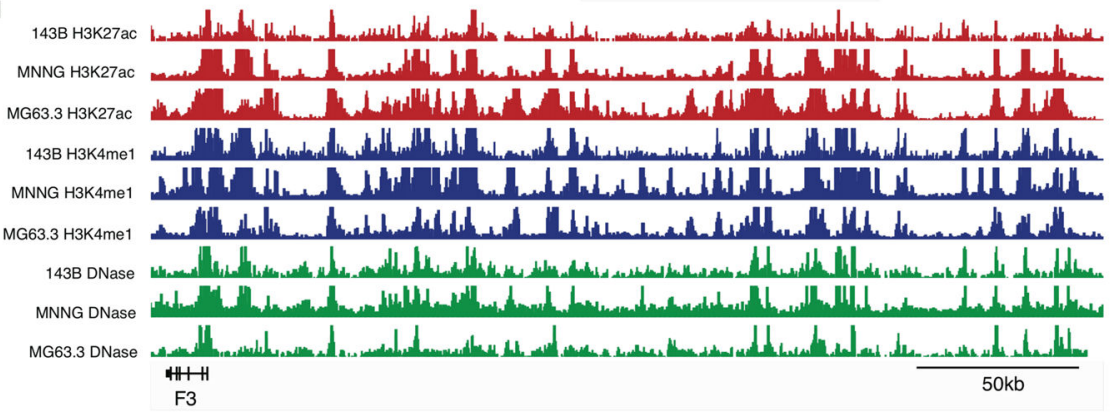

Extended Data Figure 8. Assessment of Tissue Factor (F3) dysregulation in metastatic osteosarcoma

a. IGV browser view of $\mathrm{H} 3 \mathrm{~K} 27 \mathrm{ac}, \mathrm{H} 3 \mathrm{~K} 4 \mathrm{me} 1$, and DNase profiles at $F 3$ gained Met-VEL cluster locus in MG63.3 (metastatic) and MG63 (parental) cell lines. Top of figure shows local contact profile analysis of F3 locus in MG63.3. In the top panel (main trend), the contact intensity (black line) is calculated by using a running mean analysis of normalized read counts with a $1 \mathrm{~kb}$ sliding window. The 20 th and 80 th percentile are visualized as a gray trend graph. In the bottom panel, contact intensities are computed using linearly increasing sliding windows (scaled 2-50 kb) and are displayed as a color-coded heatmap of positive 4C signals (maximum of interaction set to 1). Local color changes are log-scaled to indicate changes of statistical enrichment of captured sequences, corresponding to the enhancerpromoter interaction. Areas of significant contact highlighted.

b. Fold-change quantile normalized $F 3$ FPKM values in 3 metastatic cell lines at $24 \mathrm{hrs}$ and 14 days of metastatic outgrowth in ex vivo lung culture relative to parental line at $24 \mathrm{hrs}$. c. Tissue Factor $(F 3)$ relative expression in human patient primary tumors and lung metastases normalized to expression in normal osteoblasts.

d. IGV browser view of $\mathrm{H} 3 \mathrm{~K} 27 \mathrm{ac}, \mathrm{H} 3 \mathrm{~K} 4 \mathrm{me} 1$, and DNase profiles at F3 gained Met-VEL cluster locus in MG63.3, MNNG, and 143B metastatic cell lines.

Nat Med. Author manuscript; available in PMC 2018 July 15. 

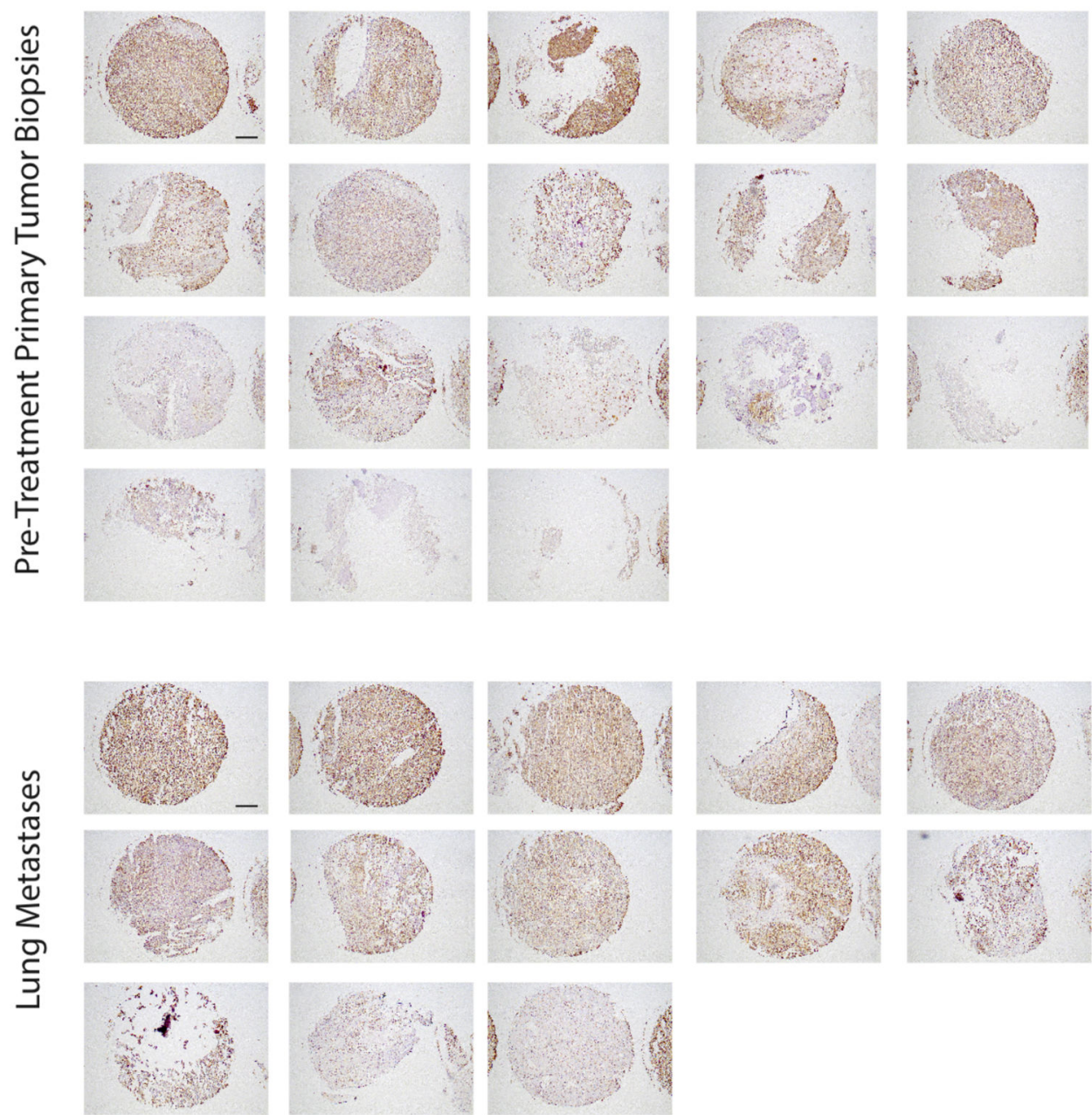

Extended Data Figure 9. F3 expression in human osteosarcoma tumors

Immunohistochemical staining of F3 in human osteosarcoma lung metastases and primary tumors. Scale bars $=200 \mu \mathrm{m}$. 
a.

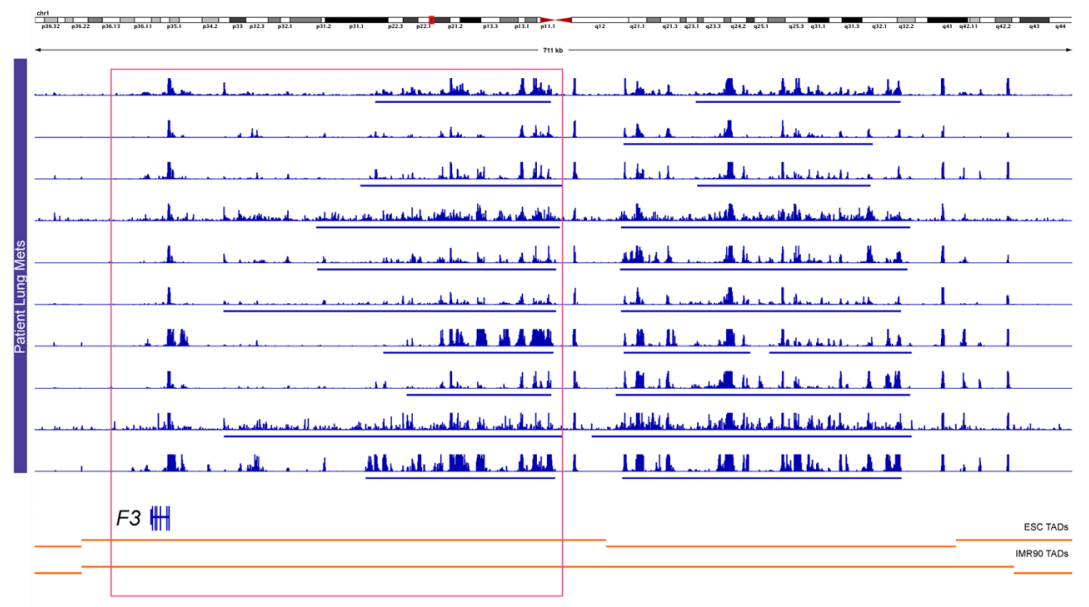

b.

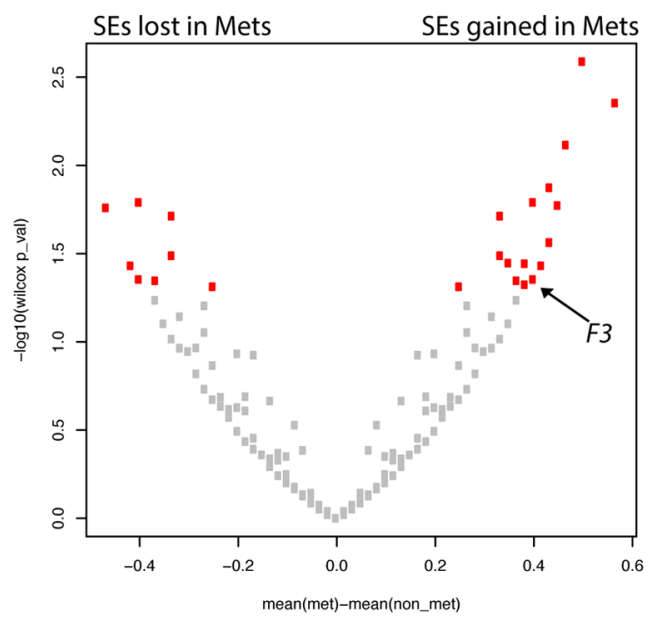

Extended Data Figure 10. Assessment of Tissue Factor (F3) Met-VELs in patient lung metastases a. IGV browser views of the $F 3$ locus showing H3K27ac ChIP-seq tracks of lung metastases from 10 osteosarcoma patients. Blue bars below each track correspond to super enhancers defined using the ROSE script. Hi-C defined topologically associating domains (TADs) are displayed below.

b. Volcano plot of 5571 total super enhancers detected in all osteosarcoma patient samples and cell lines used in this study. Points marked in red denote super enhancers meeting the threshold of significance $(\mathrm{P}<0.05)$ for being gained or lost in metastatic samples. Points in grey denote super enhancers below the significance threshold. The F3 super enhancer is indicated by the arrow. 
a
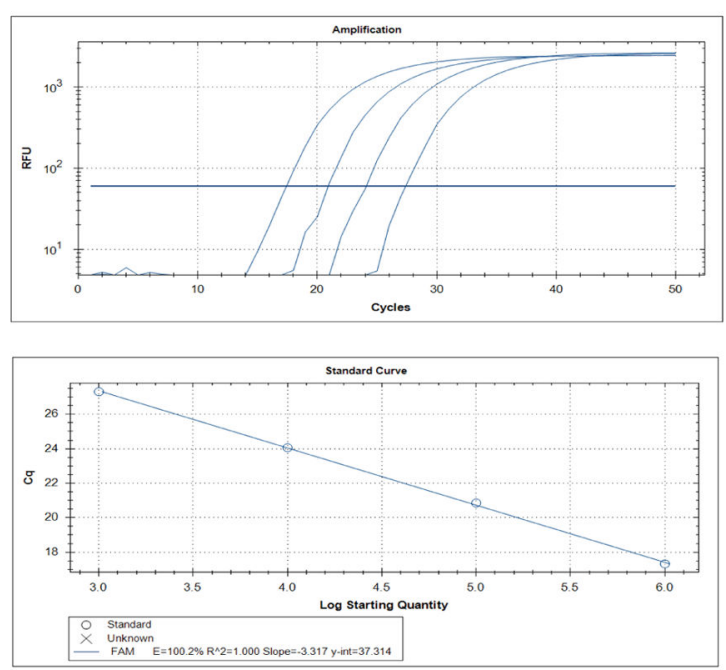

c

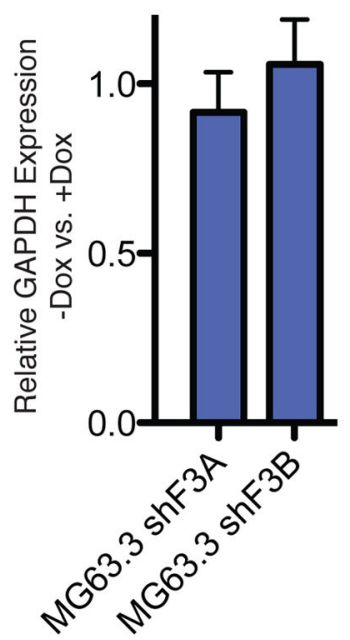

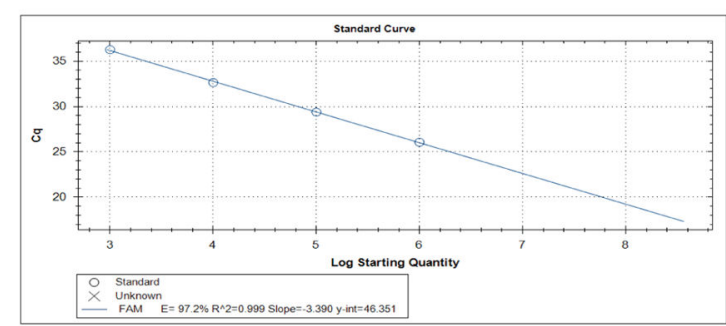

b
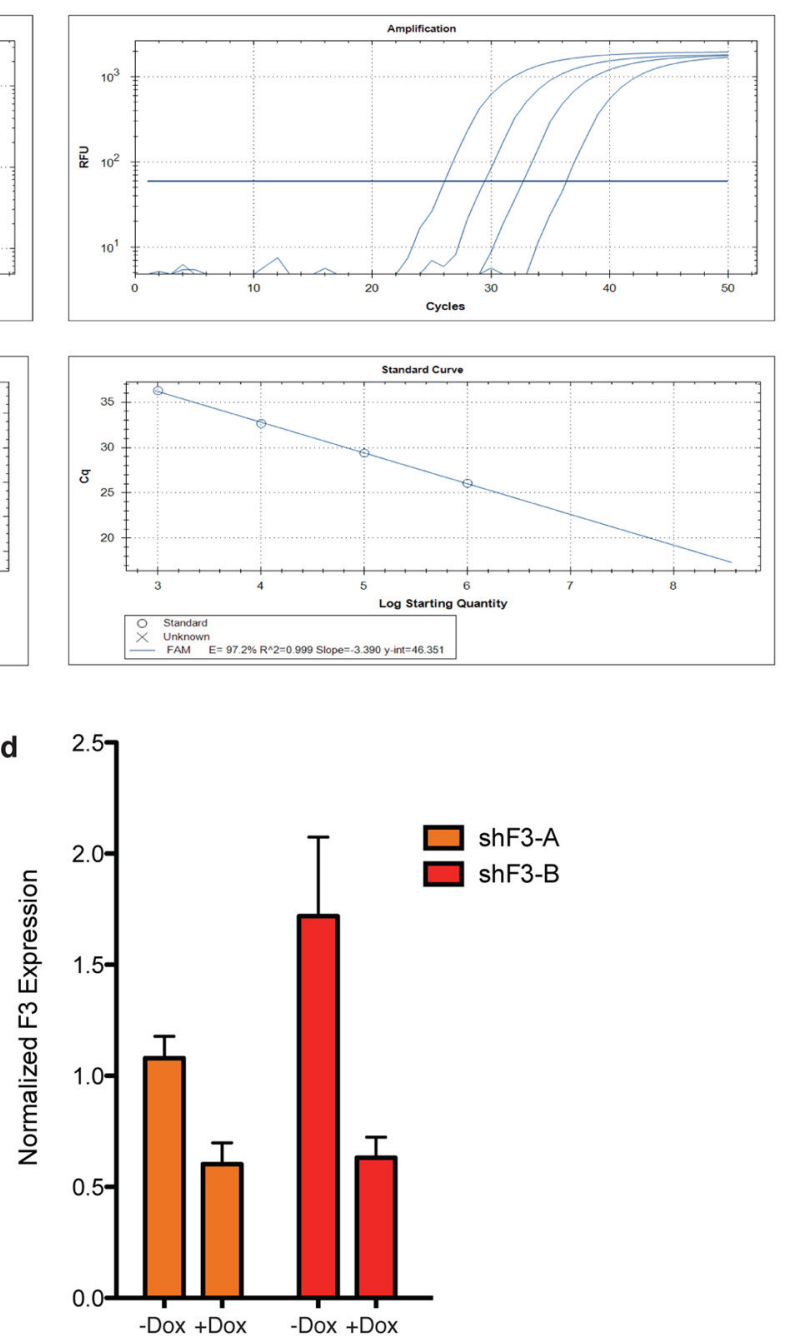

Extended Data Figure 11. Assessment of F3 knockdown by RT-qPCR

a, Amplification plots and standard curves of GAPDHRT-qPCR using 0.2ng, 2ng, 20ng, and $200 \mathrm{ng}$ of template cDNA demonstrating efficiency value of $100 \%$ and $\mathrm{R}^{2}$ value of 1.000 . All $\mathrm{Cq}$ values used for quantification of $G A P D H$ were confirmed to be within linear range of standards.

b, Amplification plots and standard curves of F3 RT-qPCR using 0.2ng, 2ng, 20ng, and 200ng of template cDNA demonstrating efficiency value of $97.2 \%$ and $\mathrm{R}^{2}$ value of 0.999 . All $\mathrm{Cq}$ values used for quantification of $F 3$ were confirmed to be within linear range of standards.

c, Relative $G A P D H$ expression in DsRed+ (induced) cells transduced with F3 shRNA constructs after $40 \mathrm{hrs}$ treatment with $5 \mathrm{ug} / \mathrm{ml}$ doxycycline relative to uninduced controls (average of 3 replicates $+/-$ SEM). 
d, Relative $F 3$ expression normalized to $G A P D H$ in DsRed+ (induced) cells transduced with F3 shRNA constructs after $40 \mathrm{hrs}$ treatment with $5 \mathrm{ug} / \mathrm{ml}$ doxycycline relative to uninduced controls (average of 3 replicates $+/$-SEM).

a
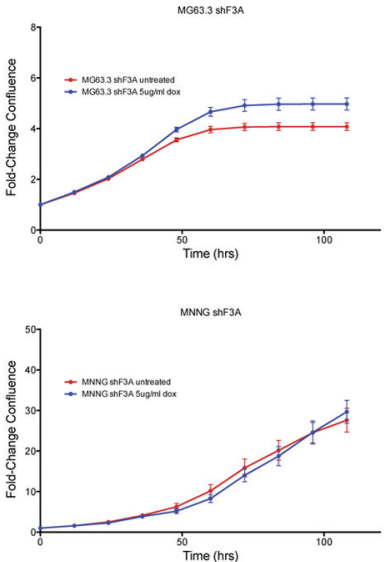

$\Rightarrow$

b

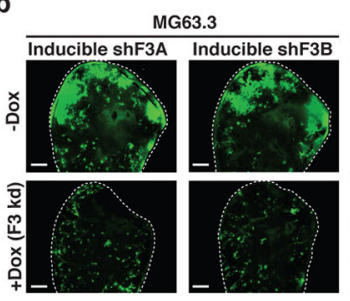

C $\quad \mathrm{MG} 63.3$

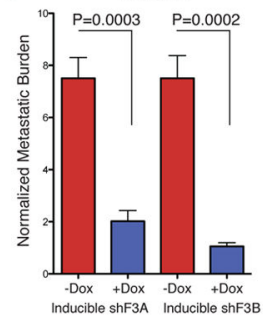

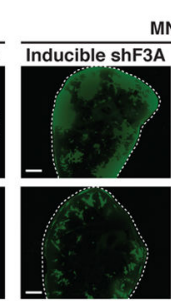

MNNG
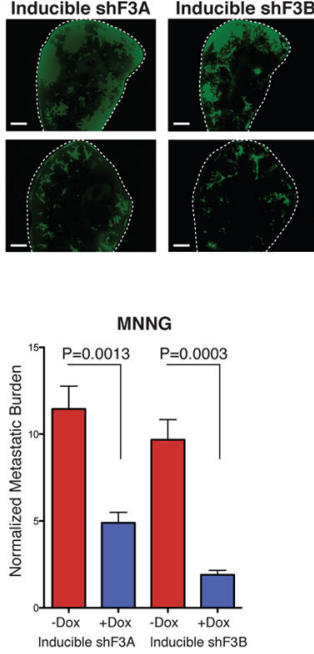

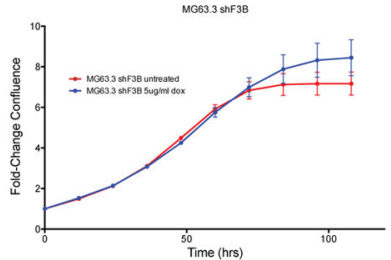

MNNG StF3B
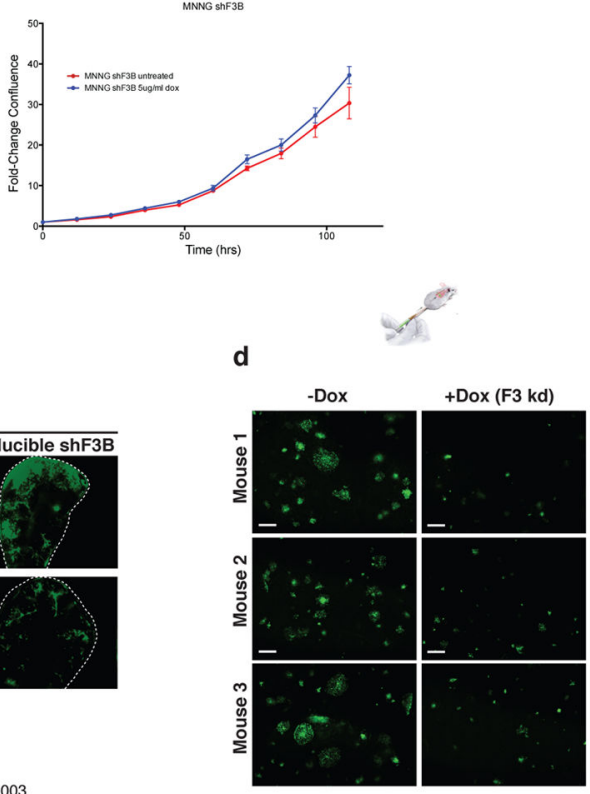

e

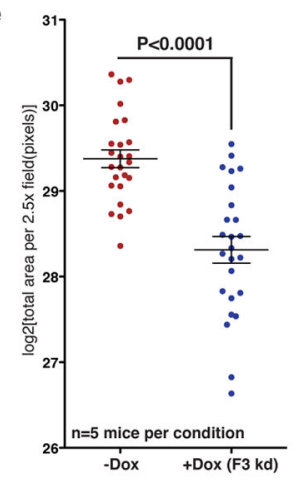

Extended Data Figure 12. Assessment of $F 3$ knockdown on metastatic osteosarcoma cell in vitro growth and lung colonization

a. Change in confluence relative to day 0 of MG63.3 (top) and MNNG (bottom) cells transduced with $F 3$ shRNA constructs grown in standard culture conditions over 108hrs in the presence or absence of $5 \mathrm{ug} / \mathrm{ml}$ doxycycline. Values represent averages from 6 plates $+/-$ SD. 
b. Representative 2.5x images of from day 21 of ex vivo lung culture sections of GFP+ MG63.3 (left) and MNNG (right) cells transduced with F3 shRNA constructs untreated (top) or treated with $5 \mathrm{ug} / \mathrm{ml}$ doxycycline (bottom). Lung sections outlined with dashed white line. Scale bar $=500 \mu \mathrm{m}$.

c. Quantification of metastatic burden at day 21 of ex vivo lung culture sections of GFP+ MG63.3 (left) and MNNG (right) cells transduced with F3 shRNA constructs untreated (red) or treated (blue) with $5 \mathrm{ug} / \mathrm{ml}$ doxycycline. Values represent averages +/-SEM from 8 sections per condition (4 sections per mouse $\mathrm{x} 2$ mice) normalized to the same section at day 0 . P-Value calculated by Mann-Whitney Test.

d. Representative $2.5 \mathrm{x}$ images of in vivo metastatic burden in untreated (left) or doxycycline-treated (right) mice receiving tail vein injection of $5 \times 10^{5} \mathrm{GFP}+\mathrm{MG63.3}$ cells transduced with shF3B construct. Scale bar $=500 \mu \mathrm{m}$.

e. Quantification of in vivo metastatic burden in untreated (red) or doxycycline-treated (blue) mice receiving tail vein injection of $5 \times 10^{5} \mathrm{GFP}+$ MG63.3 cells transduced with shF3B construct. Values represent $\log 2$ of total GFP+ area per $2.5 \mathrm{x}$ field, black bars represent average $+/-$ SEM ( $=5$ mice per condition, 5 images per mouse). P-Value calculated by Mann-Whitney Test. 
a

옹

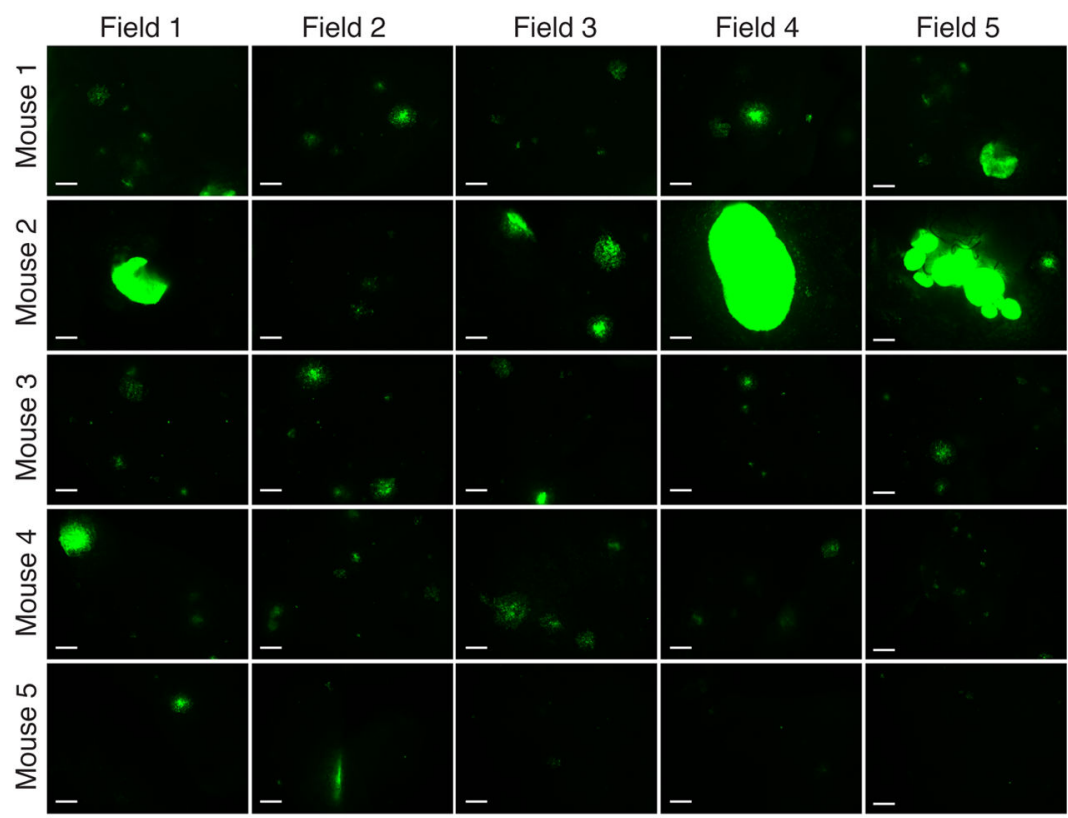

b

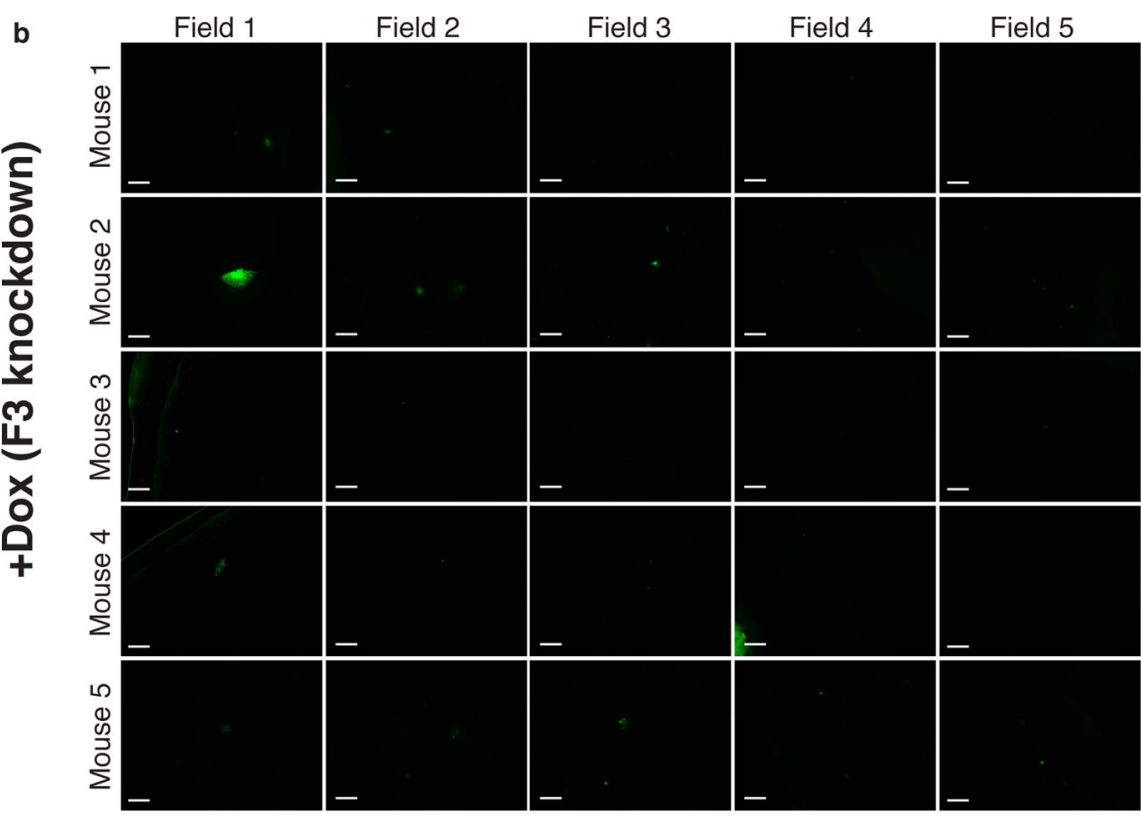

Extended Data Figure 13. Assessment of lung metastatic burden at experimental end point of orthotopic spontaneous metastasis experiment with $\boldsymbol{F} 3$ knockdown

a. All images used for quantification of in vivo metastatic lesions in lungs 21 days after measureable tumor formation in untreated mice receiving orthotopic injection of $8 \times 10^{5}$ MG63.3 cells transduced with shF3B construct ( 5 mice per condition, 5 images per mouse). Scale bar $=250 \mu \mathrm{m}$.

b. All images used for quantification of in vivo metastatic lesions in lungs 21 days after measureable tumor formation in doxycycline-treated mice receiving orthotopic injection of $8 \times 10^{5}$ MG63.3 cells transduced with shF3B construct ( 5 mice per condition, 5 images per mouse). Scale bar $=250 \mu \mathrm{m}$. 


\section{Extended Data Table 1}

Candidate metastasis dependency genes identified by in vivo high-throughput RNAi functional assay.

\begin{tabular}{|l|l|c|}
\hline Gene & Inclusion Criteria & Metastasis Specific? \\
\hline BACH2 & Enriched Motif & $\mathrm{N}$ \\
\hline DOCK4 & $\begin{array}{l}\text { Common Gained Met-VEL } \\
\text { Gained Met-VEL Cluster (MG63.3) }\end{array}$ & $\mathrm{N}$ \\
\hline F3 & Gained Met-VEL Cluster (MG63.3) & $\mathrm{Y}$ \\
\hline FBXO42 & Gained Met-VEL Cluster (MNNG/143B) & $\mathrm{Y}$ \\
\hline FLNA & $\begin{array}{l}\text { Common Gained Met-VEL } \\
\text { Gained Met-VEL Cluster (MNNG/143B) }\end{array}$ & $\mathrm{Y}$ \\
\hline FOS & Enriched Motif & $\mathrm{Y}$ \\
\hline FOSL1 & Enriched Motif & $\mathrm{Y}$ \\
\hline FOXO3 & Gained Met-VEL Cluster (MNNG/143B) & $\mathrm{Y}$ \\
\hline GHR & Gained Met-VEL Cluster (MG63.3) & $\mathrm{N}$ \\
\hline JUN & Enriched Motif & $\mathrm{N}$ \\
\hline JUNB & Enriched Motif & $\mathrm{N}$ \\
\hline RASSF2 & Common Gained Met-VEL & $\mathrm{N}$ \\
\hline TMEM230 & Common Gained Met-VEL & $\mathrm{N}$ \\
\hline
\end{tabular}

\section{Supplementary Material}

Refer to Web version on PubMed Central for supplementary material.

\section{Acknowledgments}

The authors thank the members of the Tesar, Kaplan, and Helman laboratories for their input throughout the course of the project as well as Brennan Decker for his input on the manuscript text. Additional support was provided by the Genomics Core Facility of the CWRU School of Medicine's Genetics and Genome Sciences Department and the Case Comprehensive Cancer Center (P30CA043703). This work was supported by the Liddy Shriver Sarcoma Initiative (P.C.S., C.K., J.J.M.), the QuadW Foundation (P.C.S.), the Sarcoma Foundation of America (P.C.S.), F30 CA186633 (J.J.M.), T32 GM007250 (J.J.M.), R01CA193677 (P.C.S.), R01CA204279 (P.C.S.), R01CA160356 (P.C.S.), the NIH Intramural Visiting Fellow Program \#15335 (M.M.L.), and the NIH Intramural Research Program (C. K.).

\section{References}

1. Valastyan S, Weinberg RA. Tumor metastasis: molecular insights and evolving paradigms. Cell. 2011; 147:275-292. DOI: 10.1016/j.cell.2011.09.024 [PubMed: 22000009]

2. Chambers AF, Groom AC, MacDonald IC. Dissemination and growth of cancer cells in metastatic sites. Nature reviews. Cancer. 2002; 2:563-572. DOI: 10.1038/nrc865 [PubMed: 12154349]

3. Gundem G, et al. The evolutionary history of lethal metastatic prostate cancer. Nature. 2015; 520:353-357. DOI: 10.1038/nature14347 [PubMed: 25830880]

4. Hong MK, Macintyre G, Wedge DC, Van Loo P. Tracking the origins and drivers of subclonal metastatic expansion in prostate cancer. Nature communications. 2015; 6:6605.

5. Bos PD, et al. Genes that mediate breast cancer metastasis to the brain. Nature. 2009; 459:10051009. DOI: 10.1038/nature08021 [PubMed: 19421193] 
6. Kang Y, et al. A multigenic program mediating breast cancer metastasis to bone. Cancer cell. 2003; 3:537-549. [PubMed: 12842083]

7. Minn AJ, et al. Genes that mediate breast cancer metastasis to lung. Nature. 2005 ; 436:518-524. DOI: 10.1038/nature03799 [PubMed: 16049480]

8. Factor DC, et al. Epigenomic comparison reveals activation of "seed" enhancers during transition from naive to primed pluripotency. Cell stem cell. 2014; 14:854-863. DOI: 10.1016/j.stem. 2014.05.005 [PubMed: 24905169]

9. Gifford CA, et al. Transcriptional and epigenetic dynamics during specification of human embryonic stem cells. Cell. 2013; 153:1149-1163. DOI: 10.1016/j.cell.2013.04.037 [PubMed: 23664763]

10. Zhu J, et al. Genome-wide chromatin state transitions associated with developmental and environmental cues. Cell. 2013; 152:642-654. DOI: 10.1016/j.cell.2012.12.033 [PubMed: 23333102]

11. Heintzman ND, et al. Histone modifications at human enhancers reflect global cell-type-specific gene expression. Nature. 2009; 459:108-112. DOI: 10.1038/nature07829 [PubMed: 19295514]

12. Akhtar-Zaidi B, et al. Epigenomic enhancer profiling defines a signature of colon cancer. Science (New York, NY). 2012; 336:736-739. DOI: 10.1126/science.1217277

13. Cohen AJ, et al. Hotspots of aberrant enhancer activity punctuate the colorectal cancer epigenome. Nature communications. 2017; 8:14400.

14. Hnisz D, et al. Super-enhancers in the control of cell identity and disease. Cell. 2013; 155:934947. DOI: 10.1016/j.cell.2013.09.053 [PubMed: 24119843]

15. Loven J, et al. Selective inhibition of tumor oncogenes by disruption of super-enhancers. Cell. 2013; 153:320-334. DOI: 10.1016/j.cell.2013.03.036 [PubMed: 23582323]

16. Ramaswamy S, Ross KN, Lander ES, Golub TR. A molecular signature of metastasis in primary solid tumors. Nat Genet. 2003; 33:49-54. DOI: 10.1038/ng1060 [PubMed: 12469122]

17. McDonald OG, et al. Epigenomic reprogramming during pancreatic cancer progression links anabolic glucose metabolism to distant metastasis. Nat Genet. 2017; 49:367-376. DOI: 10.1038/ng.3753 [PubMed: 28092686]

18. Kansara M, Teng MW, Smyth MJ, Thomas DM. Translational biology of osteosarcoma. Nature reviews. Cancer. 2014; 14:722-735. DOI: 10.1038/nrc3838 [PubMed: 25319867]

19. Huang YM, Hou CH, Hou SM, Yang RS. The metastasectomy and timing of pulmonary metastases on the outcome of osteosarcoma patients. Clinical medicine Oncology. 2009; 3:99-105. [PubMed: 20689616]

20. Whyte WA, et al. Master transcription factors and mediator establish super-enhancers at key cell identity genes. Cell. 2013; 153:307-319. DOI: 10.1016/j.cell.2013.03.035 [PubMed: 23582322]

21. Ren L, et al. Characterization of the metastatic phenotype of a panel of established osteosarcoma cells. Oncotarget. 2015

22. Zentner GE, Tesar PJ, Scacheri PC. Epigenetic signatures distinguish multiple classes of enhancers with distinct cellular functions. Genome research. 2011; 21:1273-1283. DOI: 10.1101/gr. 122382.111 [PubMed: 21632746]

23. Rada-Iglesias A, et al. A unique chromatin signature uncovers early developmental enhancers in humans. Nature. 2011; 470:279-283. DOI: 10.1038/nature09692 [PubMed: 21160473]

24. Huang H, Bhat A, Woodnutt G, Lappe R. Targeting the ANGPT-TIE2 pathway in malignancy. Nature reviews. Cancer. 2010; 10:575-585. DOI: 10.1038/nrc2894 [PubMed: 20651738]

25. Clayton PE, Banerjee I, Murray PG, Renehan AG. Growth hormone, the insulin-like growth factor axis, insulin and cancer risk. Nat Rev Endocrinol. 2011; 7:11-24. DOI: 10.1038/nrendo.2010.171 [PubMed: 20956999]

26. Pinski J, et al. Inhibition of growth of human osteosarcomas by antagonists of growth hormonereleasing hormone. J Natl Cancer Inst. 1995; 87:1787-1794. [PubMed: 7473836]

27. Li N, et al. Phosphodiesterase 10A: a novel target for selective inhibition of colon tumor cell growth and beta-catenin-dependent TCF transcriptional activity. Oncogene. 2015; 34:1499-1509. DOI: 10.1038/onc.2014.94 [PubMed: 24704829] 
28. van den Berg YW, Osanto S, Reitsma PH, Versteeg HH. The relationship between tissue factor and cancer progression: insights from bench and bedside. Blood. 2012; 119:924-932. DOI: 10.1182/ blood-2011-06-317685 [PubMed: 22065595]

29. Mendoza A, et al. Modeling metastasis biology and therapy in real time in the mouse lung. The Journal of clinical investigation. 2010; 120:2979-2988. DOI: 10.1172/jci40252 [PubMed: 20644255]

30. Corradin O, et al. Combinatorial effects of multiple enhancer variants in linkage disequilibrium dictate levels of gene expression to confer susceptibility to common traits. Genome research. 2014; 24:1-13. DOI: 10.1101/gr.164079.113 [PubMed: 24196873]

31. Leaner VD, et al. Inhibition of AP-1 transcriptional activity blocks the migration, invasion, and experimental metastasis of murine osteosarcoma. The American journal of pathology. 2009; 174:265-275. DOI: 10.2353/ajpath.2009.071006 [PubMed: 19074613]

32. Filippakopoulos P, et al. Selective inhibition of BET bromodomains. Nature. 2010; 468:10671073. DOI: 10.1038/nature09504 [PubMed: 20871596]

33. Lamoureux F, et al. Selective inhibition of BET bromodomain epigenetic signalling interferes with the bone-associated tumour vicious cycle. Nature communications. 2014; 5:3511.

34. Puissant A, et al. Targeting MYCN in neuroblastoma by BET bromodomain inhibition. Cancer discovery. 2013; 3:308-323. DOI: 10.1158/2159-8290.cd-12-0418 [PubMed: 23430699]

35. Bandopadhayay P, et al. BET bromodomain inhibition of MYC-amplified medulloblastoma. Clinical cancer research: an official journal of the American Association for Cancer Research. 2014; 20:912-925. DOI: 10.1158/1078-0432.ccr-13-2281 [PubMed: 24297863]

36. Fellmann C, et al. An optimized microRNA backbone for effective single-copy RNAi. Cell reports. 2013; 5:1704-1713. DOI: 10.1016/j.celrep.2013.11.020 [PubMed: 24332856]

37. Versteeg $\mathrm{HH}$, et al. Inhibition of tissue factor signaling suppresses tumor growth. Blood. 2008; 111:190-199. DOI: 10.1182/blood-2007-07-101048 [PubMed: 17901245]

38. You JS, Jones PA. Cancer genetics and epigenetics: two sides of the same coin? Cancer cell. 2012; 22:9-20. DOI: 10.1016/j.ccr.2012.06.008 [PubMed: 22789535]

39. Jones $\mathrm{S}$, et al. Comparative lesion sequencing provides insights into tumor evolution. Proc Natl Acad Sci U S A. 2008; 105:4283-4288. DOI: 10.1073/pnas.0712345105 [PubMed: 18337506]

40. Liu W, et al. Copy number analysis indicates monoclonal origin of lethal metastatic prostate cancer. Nat Med. 2009; 15:559-565. DOI: 10.1038/nm.1944 [PubMed: 19363497]

41. Campbell PJ, et al. The patterns and dynamics of genomic instability in metastatic pancreatic cancer. Nature. 2010; 467:1109-1113. DOI: 10.1038/nature09460 [PubMed: 20981101]

42. Yachida S, et al. Distant metastasis occurs late during the genetic evolution of pancreatic cancer. Nature. 2010; 467:1114-1117. DOI: 10.1038/nature09515 [PubMed: 20981102]

43. Navin N, et al. Tumour evolution inferred by single-cell sequencing. Nature. 2011; 472:90-94. DOI: 10.1038/nature09807 [PubMed: 21399628]

44. Moelans CB, et al. Genomic evolution from primary breast carcinoma to distant metastasis: Few copy number changes of breast cancer related genes. Cancer Lett. 2014; 344:138-146. DOI: 10.1016/j.canlet.2013.10.025 [PubMed: 24184827]

45. Kerbel RS, Frost P, Liteplo R, Carlow DA, Elliott BE. Possible epigenetic mechanisms of tumor progression: induction of high-frequency heritable but phenotypically unstable changes in the tumorigenic and metastatic properties of tumor cell populations by 5-azacytidine treatment. J Cell Physiol Suppl. 1984; 3:87-97. [PubMed: 6205005]

46. Rodenhiser DI. Epigenetic contributions to cancer metastasis. Clinical \& experimental metastasis. 2009; 26:5-18. DOI: 10.1007/s10585-008-9166-2 [PubMed: 18386135]

47. Javaid $S$, et al. Dynamic chromatin modification sustains epithelial-mesenchymal transition following inducible expression of Snail-1. Cell reports. 2013; 5:1679-1689. DOI: 10.1016/j.celrep. 2013.11.034 [PubMed: 24360956]

48. Latil M, et al. Cell-Type-Specific Chromatin States Differentially Prime Squamous Cell Carcinoma Tumor-Initiating Cells for Epithelial to Mesenchymal Transition. Cell stem cell. 2017; 20:191204.e195. DOI: 10.1016/j.stem.2016.10.018 [PubMed: 27889319]

49. Denny SK, et al. Nfib Promotes Metastasis through a Widespread Increase in Chromatin Accessibility. Cell. 2016; 166:328-342. DOI: 10.1016/j.cell.2016.05.052 [PubMed: 27374332] 
50. Roe JS, et al. Enhancer Reprogramming Promotes Pancreatic Cancer Metastasis. Cell. 2017; 170:875-888.e820. DOI: 10.1016/j.cell.2017.07.007 [PubMed: 28757253]

51. Khanna C, et al. An orthotopic model of murine osteosarcoma with clonally related variants differing in pulmonary metastatic potential. Clinical \& experimental metastasis. 2000; 18:261271. [PubMed: 11315100]

52. Schmidt D, et al. ChIP-seq: using high-throughput sequencing to discover protein-DNA interactions. Methods (San Diego, Calif). 2009; 48:240-248. DOI: 10.1016/j.ymeth.2009.03.001

53. Langmead B, Trapnell C, Pop M, Salzberg SL. Ultrafast and memory-efficient alignment of short DNA sequences to the human genome. Genome biology. 2009; 10:R25. [PubMed: 19261174]

54. Li H, et al. The Sequence Alignment/Map format and SAMtools. Bioinformatics (Oxford, England). 2009; 25:2078-2079. DOI: 10.1093/bioinformatics/btp352

55. Zhang Y, et al. Model-based analysis of ChIP-Seq (MACS). Genome biology. 2008; 9:R137. [PubMed: 18798982]

56. Trapnell C, Pachter L, Salzberg SL. TopHat: discovering splice junctions with RNA-Seq. Bioinformatics (Oxford, England). 2009; 25:1105-1111. DOI: 10.1093/bioinformatics/btp120

57. Trapnell C, et al. Transcript assembly and quantification by RNA-Seq reveals unannotated transcripts and isoform switching during cell differentiation. Nature biotechnology. 2010; 28:511515. DOI: $10.1038 /$ nbt.1621

58. Ramskold D, Wang ET, Burge CB, Sandberg R. An abundance of ubiquitously expressed genes revealed by tissue transcriptome sequence data. PLoS computational biology. 2009; 5:e1000598. [PubMed: 20011106]

59. McLean CY, et al. GREAT improves functional interpretation of cis-regulatory regions. Nature biotechnology. 2010; 28:495-501. DOI: 10.1038/nbt.1630

60. Reimand J, Arak T, Vilo J. g:Profiler--a web server for functional interpretation of gene lists (2011 update). Nucleic Acids Res. 2011; 39:W307-315. DOI: 10.1093/nar/gkr378 [PubMed: 21646343]

61. Merico D, Isserlin R, Stueker O, Emili A, Bader GD. Enrichment map: a network-based method for gene-set enrichment visualization and interpretation. PLoS One. 2010; 5:e13984. [PubMed: 21085593]

62. Song L, Crawford GE. DNase-seq: a high-resolution technique for mapping active gene regulatory elements across the genome from mammalian cells. Cold Spring Harbor protocols. 2010; 2010 pdb.prot5384.

63. van de Werken HJ, et al. Robust 4C-seq data analysis to screen for regulatory DNA interactions. Nature methods. 2012; 9:969-972. DOI: 10.1038/nmeth.2173 [PubMed: 22961246]

64. Liu T, et al. Cistrome: an integrative platform for transcriptional regulation studies. Genome biology. 2011; 12:R83. [PubMed: 21859476]

65. Knott SR, et al. A computational algorithm to predict shRNA potency. Molecular cell. 2014; 56:796-807. DOI: 10.1016/j.molcel.2014.10.025 [PubMed: 25435137]

66. Zuber J, et al. Toolkit for evaluating genes required for proliferation and survival using tetracycline-regulated RNAi. Nature biotechnology. 2011; 29:79-83. DOI: 10.1038/nbt.1720

67. Goecks J, Nekrutenko A, Taylor J. Galaxy: a comprehensive approach for supporting accessible, reproducible, and transparent computational research in the life sciences. Genome biology. 2010; 11:R86. [PubMed: 20738864]

68. Osborne TS, et al. Evaluation of eIF4E expression in an osteosarcoma-specific tissue microarray. Journal of pediatric hematology/oncology. 2011; 33:524-528. DOI: 10.1097/MPH. 0b013e318223d0c1 [PubMed: 21941146]

69. Sakuma T, et al. Efficient TALEN construction and evaluation methods for human cell and animal applications. Genes Cells. 2013; 18:315-326. DOI: 10.1111/gtc.12037 [PubMed: 23388034]

70. Cermak T, et al. Efficient design and assembly of custom TALEN and other TAL effector-based constructs for DNA targeting. Nucleic Acids Res. 2011; 39:e82. [PubMed: 21493687] 
a

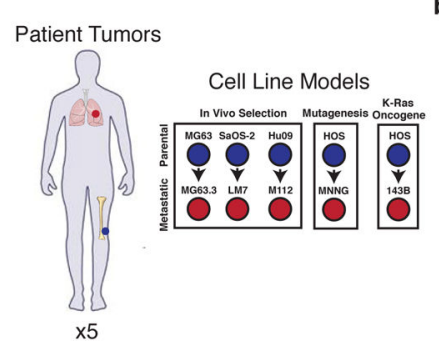

d

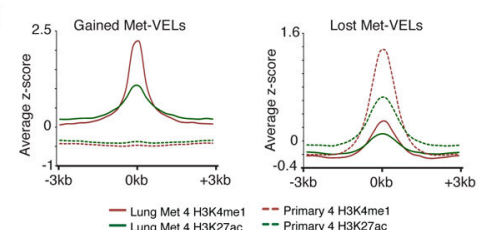

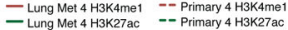

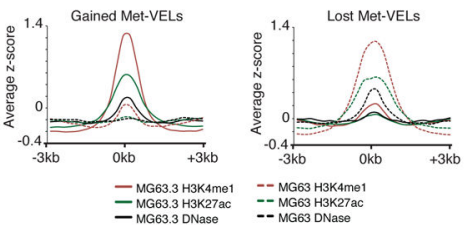

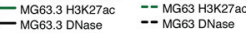

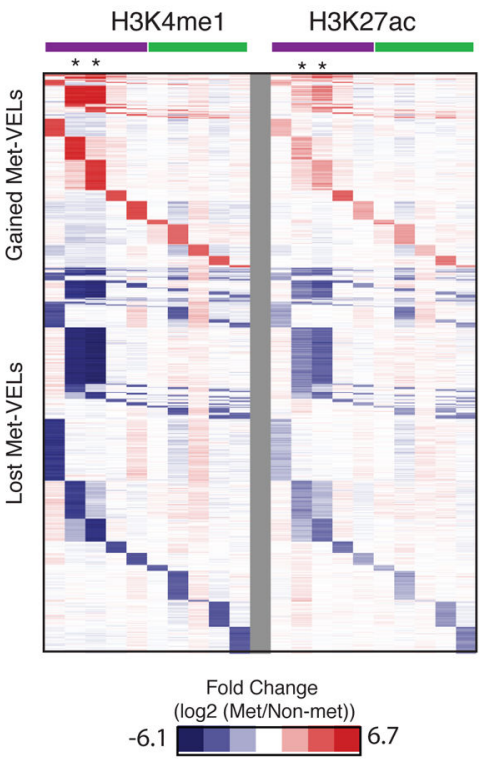

c

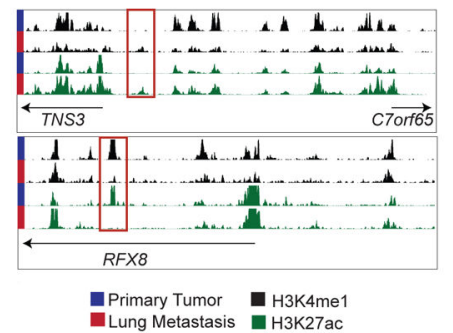

Lung Metastasis H3K27ac
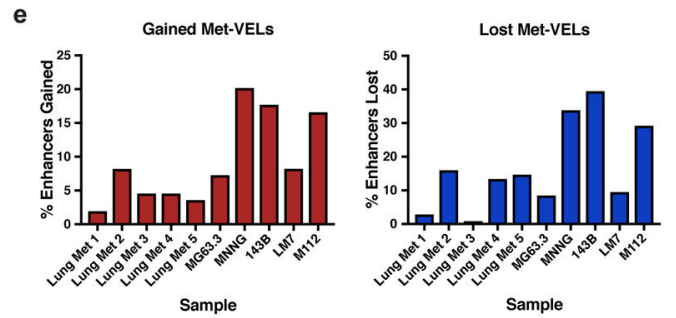

g Patient Lung Metastasis

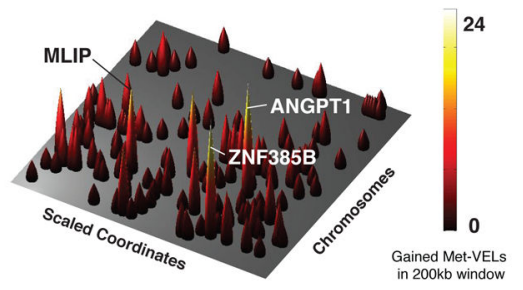

Cell Line

Patient Tumor

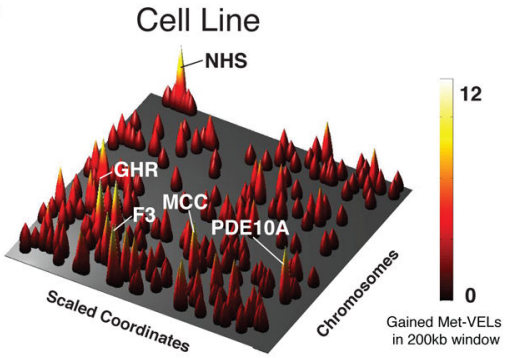

Figure 1. H3K4me1 ChIP-seq identifies metastatic variant enhancer loci (Met-VELs) and MetVEL clusters

a, Schematic representation of human tumor and metastatic human osteosarcoma cell line cohort.

b, UCSC browser views of H3K4me1 profiles from MG63.3 (metastatic) and MG63 (parental) cell lines illustrating an example of gained (top) and lost (bottom) Met-VEL(s). Met-VELs are boxed in red.

c, Heatmap showing H3K4me1 ChIP-seq signal +/-5kb from H3K4me1 peak midpoints for all putative enhancers in MG63.3/MG63 pair sorted by differences in signal. Sub-panel shows heatmap for gained and lost Met-VELs alone. 
d, Aggregate plots showing H3K4me1 ChIP-seq and H3K27ac ChIP-seq signal +/- 3kb from mid-points of gained (left) and lost (right) Met-VELs for a representative matched primary/lung metastatic human tumor pair (top) and MG63.3/MG63 cell line pair (bottom). DNase-seq signal $+/-3 \mathrm{~kb}$ from Met-VEL mid-points is also shown for the MG63.3 and MG63 cell lines.

e. Percentage of enhancers gained and lost in metastatic samples relative to primary tumors or non-metastatic cell lines.

f. Heatmap of fold change normalized RPKM in metastatic samples vs. primary tumor or non-metastatic cell lines for aggregated list of all gained and lost Met-VELs across all samples. H3K4me1 signal shown on the left, H3K27ac signal shown on the right. The samples from left to right are as follows M112, 143B, MNNG, MG63.3, LM7, Lung Met 1, Lung Met 2, Lung Met 3, Lung Met 4, Lung Met 5. Asterisks indicate 143B and MNNG samples.

g. Genome-wide gained Met-VEL landscape for human osteosarcoma metastatic tumor (Lung Met 4) and MG63.3 cell line. Rows represent scaled chromosomal coordinates. Peaks represent maximum gained Met-VEL counts in 200kb sliding windows. Predicted target genes for selected peaks are labeled. 

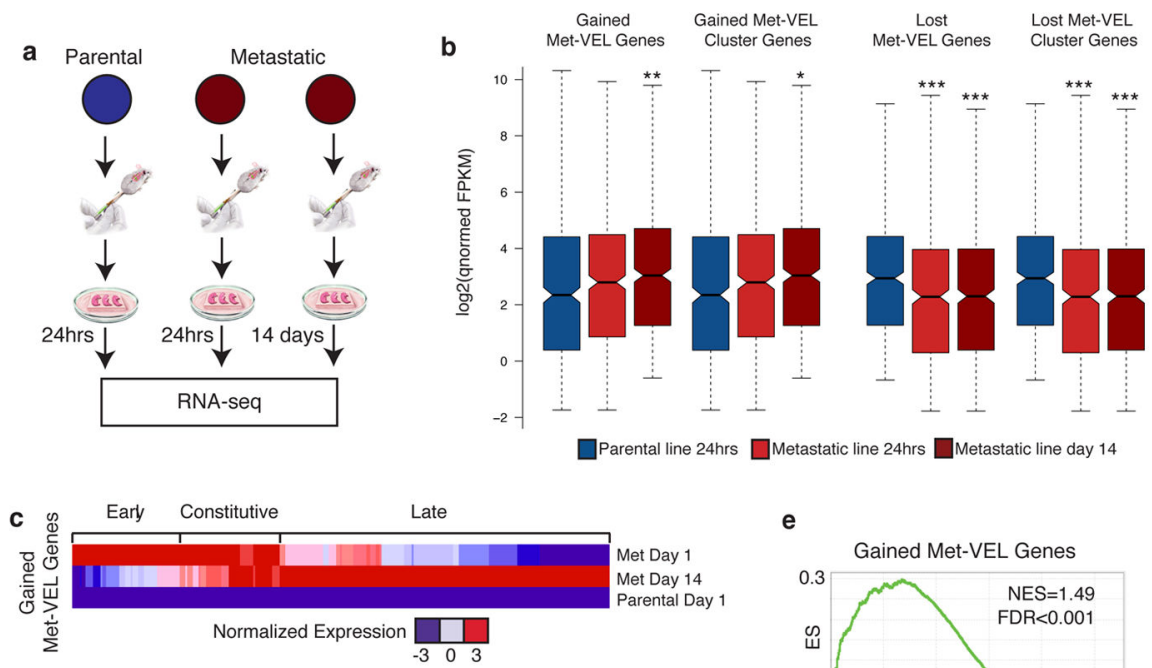

e
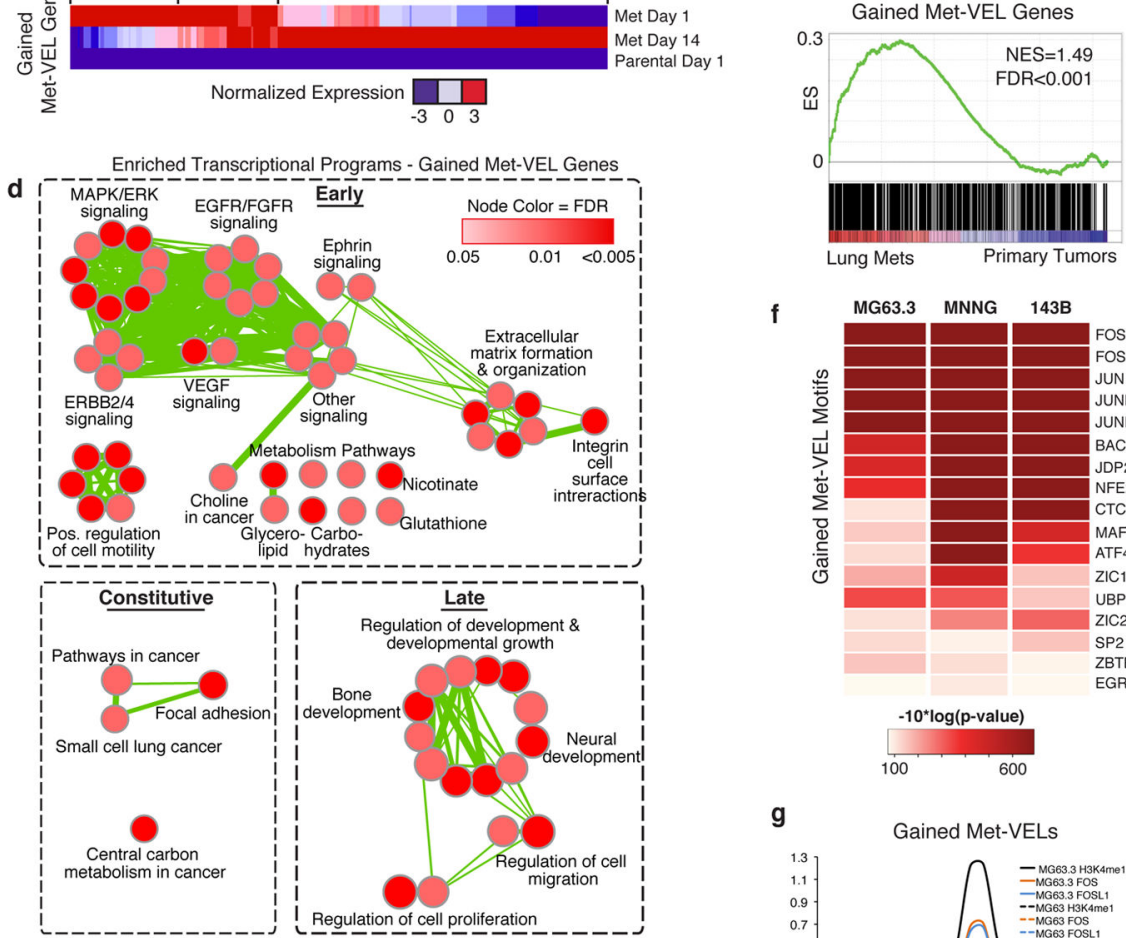

f
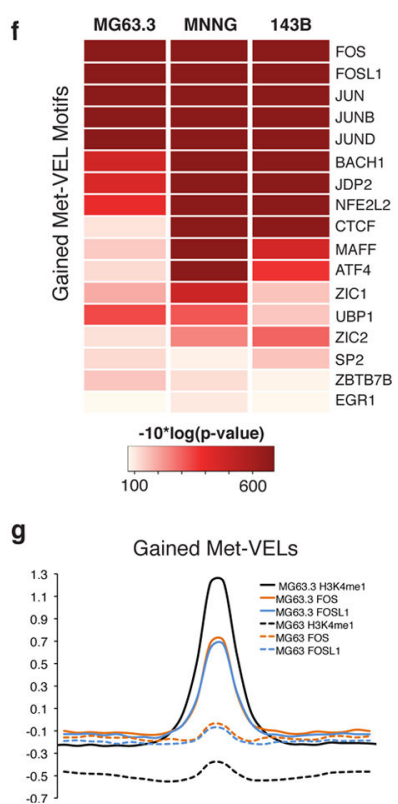

Figure 2. Met-VELs modulate gene expression during metastatic colonization of the lung a. Schematic of experimental design for assessment of Met-VEL gene expression in parental and metastatic cell lines in ex vivo lung metastasis model. Image adapted from ${ }^{29}$.

b. Log2 quantile-normalized FPKM values for gained (left) and lost (right) Met-VEL and Met-VEL cluster genes in MG63/MG63.3 cell line pair. Asterisks indicate significant differences in FPKM distributions between parental and metastatic cell lines $(* \mathrm{P}<0.05$; ** $\mathrm{P}<1 \mathrm{E}-3$; *** $\mathrm{P}<1 \mathrm{E}-4)$. P-values calculated by Mann-Whitney Test.

c. Heatmap of up-regulated gained Met-VEL genes in MG63/MG63.3 cell lines illustrating phasic expression pattern. 
d. Enriched Map representation of all Gene Ontology (GO) terms for three classes of gained Met-VEL responder genes calculated by aggregating gene lists from all three cell line pairs. e. GSEA plot of up-regulated gained Met-VEL gene set compiled from three metastatic cell lines in human patient lung metastases versus primary tumors.

f. Expressed transcription factors with enriched motifs in gained Met-VELs in three metastatic/parental cell line pairs and corresponding motif enrichment p-values.

g. Aggregate plot of H3K4me1, FOS, and FOSL1 ChiP-seq signal at all gained Met-VELs in MG63.3 cell line. 
a
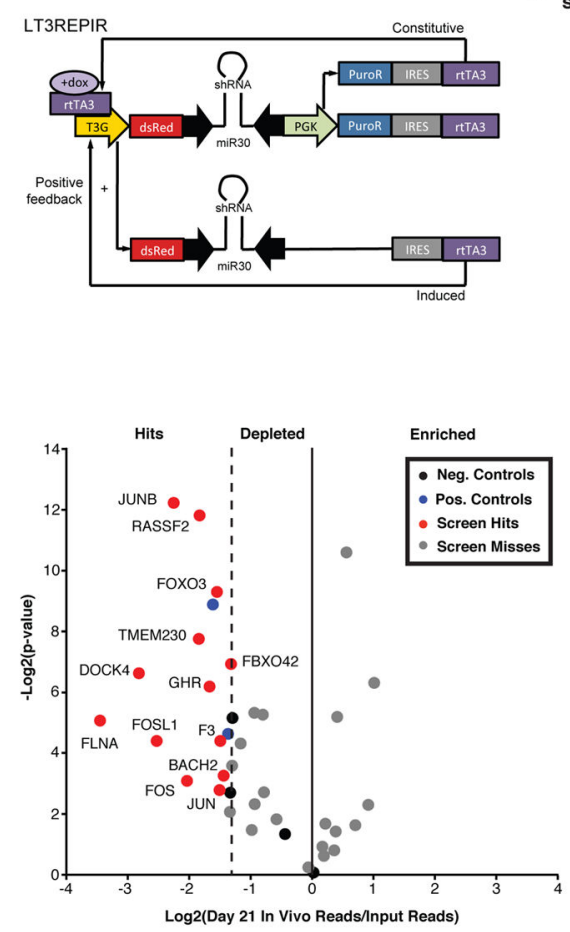

b

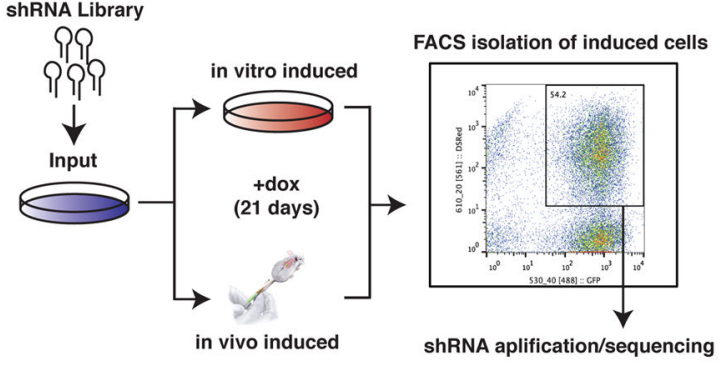

d

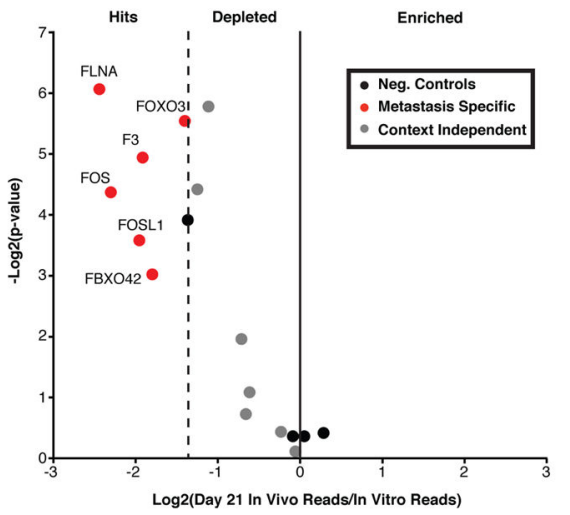

Figure 3. In Vivo high-throughput RNAi functional assay of candidate metastasis dependency genes

a. Schematic of doxycycline-inducible LT3REPIR shRNA construct. Modified from ${ }^{36}$.

b. Schematic of experimental design for in vivo high-throughput functional assay of candidate metastasis dependency genes.

c. Volcano plot of relative abundance of shRNAs targeting 33 genes in GFP+/DsRed+ sorted osteosarcoma cells from doxycycline-treated mice ( $\mathrm{N}=15$ mice per replicate $\mathrm{x} 3$ replicates) versus input cell population. $2^{\text {nd }}$ most depleted shRNAs for each gene are plotted as well as negative and positive shRNA controls. Negative controls contained groups of 2-4 shRNAs.

d. Volcano plot of relative abundance of shRNAs targeting 13 genes meeting initial hit criteria (Fig. 3c) in GFP+/DsRed+ sorted osteosarcoma cells from doxycycline-treated mice $(\mathrm{N}=5$ per replicate $\mathrm{x} 3$ replicates) versus GFP+/DsRed+ sorted osteosarcoma cells treated with doxycycline in vitro. $2^{\text {nd }}$ most depleted shRNAs for each gene are plotted as well as negative controls groups of $2-4$ shRNAs. 
a

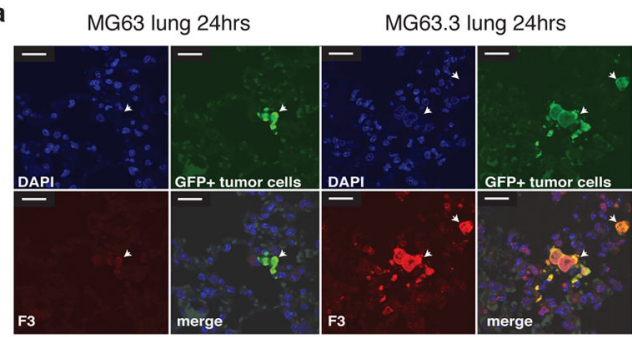

c

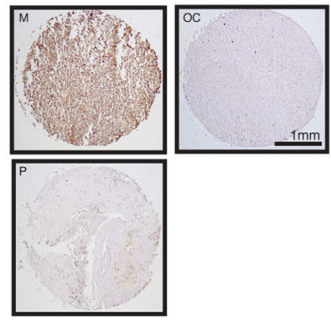

f Primary Orthotopic Tumor
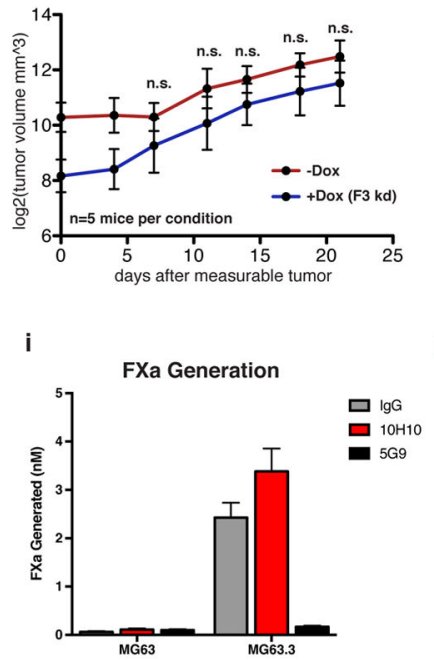

d
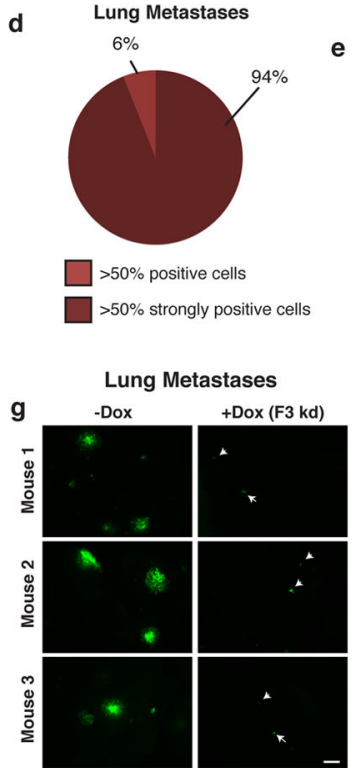

j

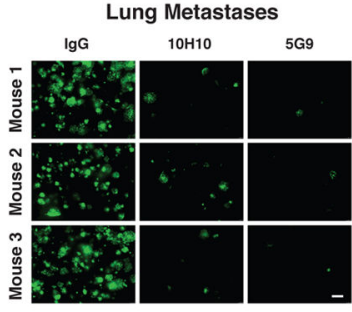

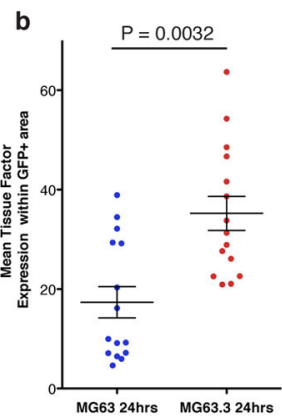

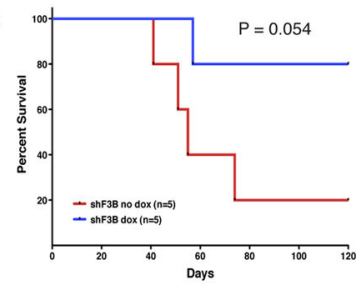

Micrometastases

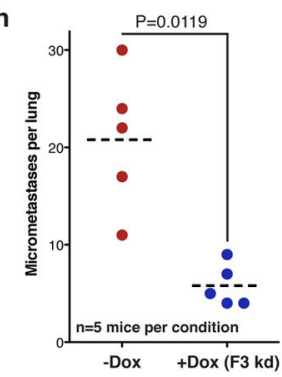

k Metastatic Burden

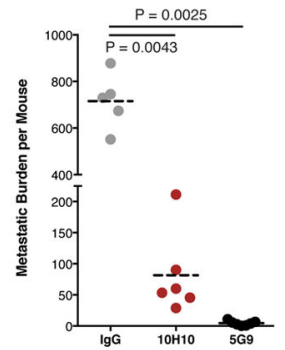

Figure 4. Tissue Factor (F3) mediates lung metastasis of osteosarcoma

a. In Vivo 2.5x images of GFP+ MG63 (parental) and MG63.3 (metastatic) cells in the lung $24 \mathrm{hrs}$ following tail-vein injection of $1 \times 10^{6}$ cells. Sections stained for GFP, tissue factor (F3, red), and DAPI. Arrowheads indicate individual tumor cells within lung. Scale bars $=20 \mu \mathrm{m}$. b. Quantification of mean red pixel intensity within GFP+ (tumor) area in MG63 (parental) and MG63.3 (metastatic) cells 24hrs after tail-vein injection ( $\mathrm{N}=15$ images per condition). c. Representative images of immunohistochemical staining of F3 in human osteosarcoma lung metastases $(\mathrm{M})$, primary tumor $(\mathrm{P})$, and omission control (OC). Tissue microarray contained 18 scoreable lung metastases of similar quality to those displayed.

d. Percentage of lung metastases with various levels of F3 positivity. 
e. Kaplan-Meier plot of untreated (red) and doxycycline-treated (blue) mice tail-vein injected with $5 \times 10^{4}$ GFP+ MG63.3 cells transduced with shF3B construct $(\mathrm{N}=5$ mice per condition). P-value calculated by Gehan-Breslow-Wilcoxon Test.

f. Primary tumor growth in untreated (red) and doxycycline-treated (blue) mice receiving orthotopic injection of $8 \times 10^{5}$ GFP+ MG63.3 cells transduced with shF3B construct. Values represent averages + /-SEM ( $\mathrm{N}=5$ mice per condition). P-values calculated using student's ttest.

g. Representative $2.5 \mathrm{x}$ images of in vivo metastatic lesions in lungs 21 days after measureable tumor formation in untreated (left) and doxycycline-treated (right) mice receiving orthotopic injection of $8 \times 10^{5} \mathrm{GFP}+\mathrm{MG} 63.3$ cells transduced with shF3B construct. Arrowheads indicate individual tumor cells within lung. Scale bar $=500 \mu \mathrm{m}$. h. Quantification of lung metastatic burden 21 days after measureable tumor formation in untreated (red) and doxycycline-treated (blue) mice receiving orthotopic injection of $8 \times 10^{5}$ GFP+ MG63.3 cells transduced with shF3B construct ( $N=5$ mice per condition, 5 images per mouse). P-values calculated using Mann-Whitney test.

i. Amount of activated factor X (FXa) formed in in vitro assay by MG63 (left) and MG63.3 (right) cells treated with $25 \mu \mathrm{g} / \mathrm{mL} \mathrm{IgG}$ control, 10H10, or 5G9 antibodies for 20 minutes prior to the addition of FVIIa and FX to a final concentration of 100nM. FXa formation assessed 30 minutes after adding FX. P-values calculated using student's t test with Welch's correction.

j. Representative $2.5 \mathrm{x}$ images of in vivo metastatic lesions in lungs of mice 14 days after tail vein injection of $5 \times 10^{5}$ MG63.3 cells with $500 \mu \mathrm{g}$ of $\mathrm{IgG}, 5 \mathrm{G} 9$, or $10 \mathrm{H} 10$ antibodies. Scale bar $=500 \mu \mathrm{m}$.

k. Quantification of lung metastatic burden 14 days after tail vein injection of $5 \times 10^{5}$

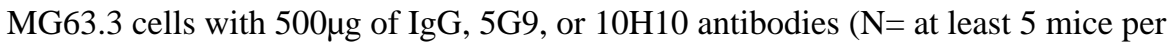
condition, 5 images per mouse). P-values calculated using Mann-Whitney test. 
a
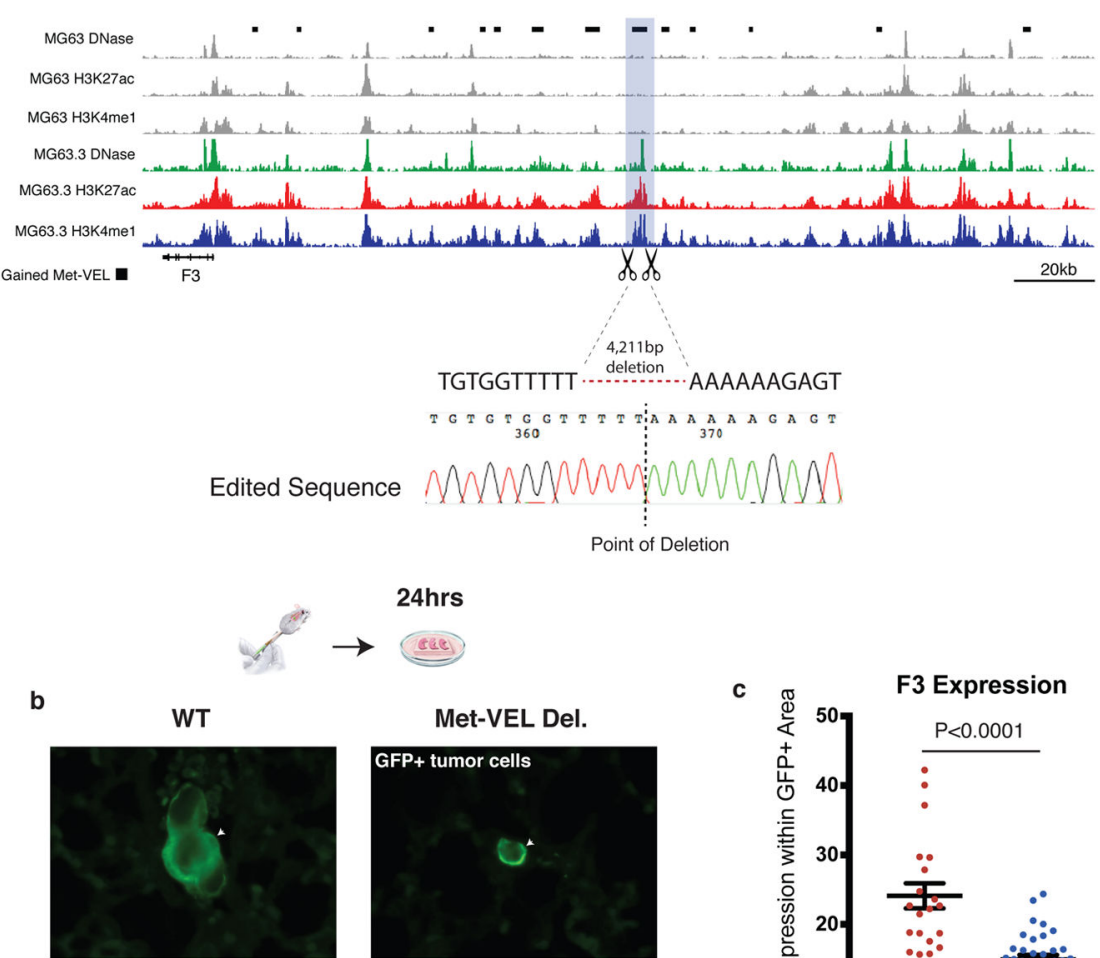

$24 \mathrm{hrs}$

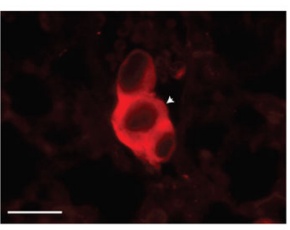

Met-VEL Del.
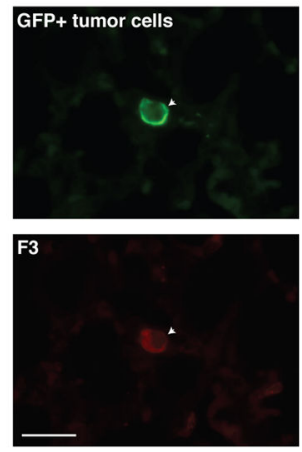

5 days

d

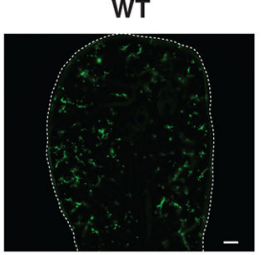

Met-VEL Del.

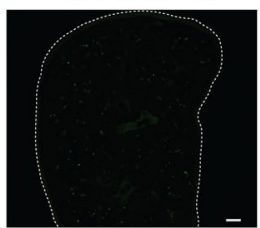

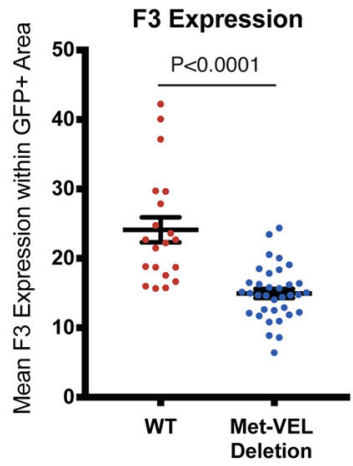

e

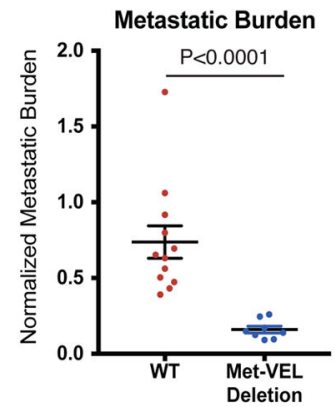

Figure 5. Deletion of single gained Met-VEL blunts F3 expression and mitigates lung metastasis of osteosarcoma cells

a. IGV browser view of region targeted for deletion with TALENs. Schematic shows strategy for 4,211bp deletion. Sanger sequencing shows resulting clonal homozygous deletion.

b. Representative 40x images of WT and Met-VEL deleted MG63.3 cells in the lung 24hrs after initiation of an ex vivo lung metastasis experiment. Scale bar $=50 \mu \mathrm{m}$.

c. Quantification of mean red pixel intensity (F3 expression) within GFP+ (tumor) area for WT and Met-VEL deleted MG63.3 cells 24hrs after initiation of ex vivo lung metastasis assay ( $\mathrm{N}=$ at least 20 images per condition, sections taken from $\mathrm{N}=3-5$ mice per condition). P-value calculated by Mann-Whitney Test. 
d. Representative 2.5x images of WT (left) and Met-VEL deleted (right) lung sections at day 5. Lung sections outlined with dashed white line. Scale bar $=500 \mu \mathrm{m}$.

e. Quantification of metastatic burden at day 5 of ex vivo lung culture for GFP+ WT and Met-VEL deleted MG63.3 cells. Bars represent mean +/-SEM from at least 8 sections per condition (4 sections per mouse x 2-3 mice) normalized to the same section at day 0 . PValue calculated by Mann-Whitney Test. 
Table 2-1

\begin{tabular}{|c|c|c|}
\hline & \multicolumn{2}{|c|}{ 4C-seq primers } \\
\hline & \multicolumn{2}{|l|}{ 4C-seq Primers: } \\
\hline & Forward Primer & CTGTAAACTTGCCAAGCATG \\
\hline$\vec{d}$ & Reverse Primer & GGTCGCACAGGAGAAAGG \\
\hline
\end{tabular}


Table 2-4

Probes used for F3RT-qPCR

\begin{tabular}{|c|c|}
\hline Gene Symbol & TaqMan Gene Expression Assay Primer \\
\hline F3 & Hs01076029_m1 \\
\hline GAPDH & Hs02758991_g1 \\
\hline
\end{tabular}

